\title{
Regulatory non-coding RNAs: a new frontier in regulation of plant biology
}

\author{
Sailaja Bhogireddy ${ }^{1}\left(\mathbb{D} \cdot\right.$ Satendra K. Mangrauthia ${ }^{2}\left(\mathbb{C}^{\circ} \cdot\right.$ Rakesh Kumar $^{1,3} \cdot$ Arun K. Pandey $^{1,4} \cdot$ Sadhana Singh $^{1}$. \\ Ankit Jain ${ }^{1} \cdot$ Hikmet Budak $^{5}$ (i) Rajeev K. Varshney ${ }^{1,6}$ (i) $\cdot$ Himabindu Kudapa ${ }^{1}$ (i)
}

Received: 24 December 2020 / Revised: 21 April 2021 / Accepted: 26 April 2021 / Published online: 20 May 2021

(c) The Author(s) 2021

\begin{abstract}
Beyond the most crucial roles of RNA molecules as a messenger, ribosomal, and transfer RNAs, the regulatory role of many non-coding RNAs (ncRNAs) in plant biology has been recognized. ncRNAs act as riboregulators by recognizing specific nucleic acid targets through homologous sequence interactions to regulate plant growth, development, and stress responses. Regulatory ncRNAs, ranging from small to long ncRNAs (lncRNAs), exert their control over a vast array of biological processes. Based on the mode of biogenesis and their function, ncRNAs evolved into different forms that include microRNAs (miRNAs), small interfering RNAs (siRNAs), miRNA variants (isomiRs), lncRNAs, circular RNAs (circRNAs), and derived ncRNAs. This article explains the different classes of ncRNAs and their role in plant development and stress responses. Furthermore, the applications of regulatory ncRNAs in crop improvement, targeting agriculturally important traits, have been discussed.
\end{abstract}

Keywords Regulatory non-coding RNAs $\cdot$ Biogenesis $\cdot$ Degradation $\cdot$ IsomiRs $\cdot$ Stress response $\cdot$ Crop improvement

\section{Introduction}

Crop plants adapt to different regulatory mechanisms to accomplish sustainable productivity. A myriad of non-coding RNAs (ncRNAs) are important players in these regulatory networks. In the recent past, research on ncRNAs

Sailaja Bhogireddy

sailajaprasadd@gmail.com

$\triangle$ Himabindu Kudapa

k.himabindu@cgiar.org

1 Center of Excellence in Genomics \& Systems Biology (CEGSB), International Crops Research Institute for the Semi-Arid Tropics (ICRISAT), Hyderabad, India

2 Crop Improvement Section, ICAR-Indian Institute of Rice Research, Hyderabad, India

3 Department of Life Sciences, Central University of Karnataka, Karnataka, India

4 College of Life Sciences, China Jiliang University, Hangzhou, China

5 Montana BioAgriculture, Inc., Missoula, MT, USA

6 State Agricultural Biotechnology Centre, Centre for Crop and Food Innovation, Murdoch University, Murdoch, Western Australia, Australia has been accelerated with the advent of deep sequencing technologies in the field of molecular biology. The ncRNAs derived from transcriptionally active genes do not encode a functional protein (Palazzo and Lee 2015). The structural class of ncRNAs comprises ribosomal RNA (rRNA), transfer RNA (tRNA), small nuclear RNA (snRNA), and small nucleolar RNA (snoRNA) (Fig. 1). The regulatory ncRNAs (rncRNAs) are broadly classified into long ncRNAs (lncRNAs, > $200 \mathrm{nt}$ ) and small ncRNAs (sncRNAs, 18-30 nt). Furthermore, several studies have reported the participation of other regulatory ncRNAs such as "circular" RNAs (circRNAs) and derived ncRNAs in plant processes (Sablok et al. 2016; Zhu et al. 2018).

The first ncRNA characterized in baker's yeast was alanine tRNA (Holley et al. 1965). The catalytic role of the RNA in the 1980s opened a new perspective for researchers to understand the complex role of different ncRNAs (Morris and Mattick 2014). Subsequently, discovery of the regulatory action of ncRNAs in Caenorhabditis elegans was emerged as a big revolution in the world of "nc" RNA and led to the identification of different classes of ncRNAs in humans, animals, and plants (Chen 2009; Lee et al. 1993; Mattick and Makunin 2006; Yu et al. 2019). Besides, technological advancements also paved the way for identification 


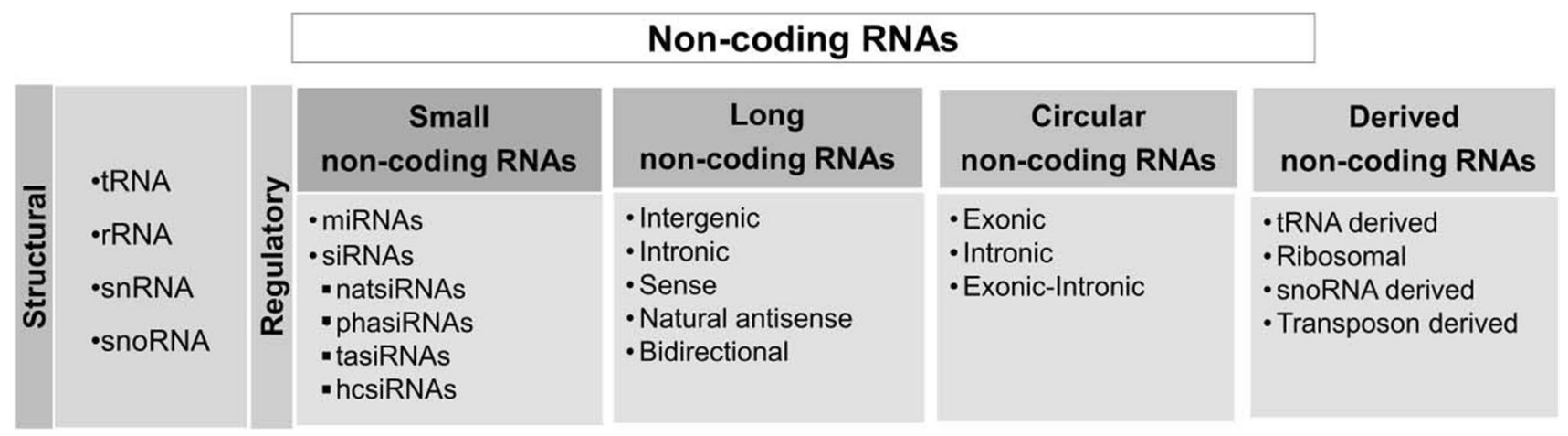

Fig. 1 Classification of non-coding RNAs: different classes and subclasses of structural and regulatory non-coding RNAs

of several other regulatory ncRNAs modulating the expression of protein-coding genes in various cellular processes by interacting with different molecular pathways. However, the gathered knowledge of ncRNAs in plants is less compared to that in animals. In this review, we present the updates on the diverse regulatory role of ncRNAs in plant biology including ncRNA variants/isoforms, circRNAs, and derived ncRNAs. Furthermore, examples of ncRNA-mediated regulation in the development of plant phenotypes with improved agronomic traits and the possible ways to utilize this information in crop improvement programs are discussed.

\section{Biogenesis}

ncRNA biogenesis is a complex phenomenon and can be derived from two major pathways: canonical and non-canonical. Canonical pathway denotes the ncRNA synthesis by classical steps (Fig. 2), while non-canonical pertains to nonclassic/non-regular ways that follow alternative pathways (Fig. 3).

\section{Canonical biogenesis}

\section{miRNAs}

Canonical biogenesis of miRNAs and other siRNAs have been well-described earlier (Borges and Martienssen 2015). In brief, the miRNA biogenesis includes the synthesis of long primary transcripts called primary miRNAs (pri-miRNAs) with the aid of RNA Polymerase (Pol) II (Pol II), and it is further processed into precursor miRNAs (pre-miRNAs/ premiR) by a set of proteins - DICER-LIKE 1 (DCL1), HYPONASTIC LEAVES 1 (HYL1), and SERRATE (SE) (Rogers and Chen 2013). Subsequent processing of premiRNA to miRNA/miRNA* duplex followed by the transfer of duplex from the nucleus to cytoplasm, where the preferential loading of mature miRNA to RNA-INDUCED
SILENCING COMPLEX (RISC) occurs and reaches its target mRNA transcript by binding with ARGONAUTE 1 (AGO 1) protein. Thus, the regulation of target gene expression occurs either by transcript cleavage or by translational repression, and the miRNA* strand gets degraded (Borges and Martienssen 2015; Budak et al. 2015). miRNAs with 21-nt length are processed by DCL1 or DCL4 proteins, while 22 and 24-nt length miRNAs are processed by DCL2 and DCL3 proteins (Fig. 2a).

\section{siRNAs}

In contrast to miRNAs, siRNAs are either exogenous or endogenous, derived from the complementary long doublestranded RNAs by RNA-DEPENDENT RNA POLYMERASE (RDR) and cleaved into siRNAs by DCL1 proteins. Different classes of endogenous siRNAs in plants include secondary siRNAs (natural antisense transcript-derived siRNAs (natsiRNAs or nat-siRNAs), phased siRNAs (phasiRNAs), and trans-acting siRNAs (tasiRNAs or ta-siRNAs)) and heterochromatic siRNAs (hcsiRNAs) (Borges and Martienssen 2015) (Fig. 2a). natsiRNAs are the pairs of perfect complementary transcripts transcribed from endogenous coding or ncRNAs with the aid of Pol II/Pol IV, RDR2, and DCL1/DCL3-dependent pathway involved in post-transcriptional gene regulation through RNA interference (RNAi) (Borges and Martienssen 2015). tasiRNAs and phasiRNAs are generated from cleavage fragments of miRNA-target transcripts, and their precursors are transcribed from noncoding loci and protein-encoding genes, respectively, by Pol II. The subsequent cleavage of transcripts by miRNA-mediated AGO1/AGO7 results in single-strand RNAs (ssRNAs) and further into double-stranded RNAs (dsRNAs) by RDR6 and SUPPRESSOR OF GENE SILENCING3 (SGS3). Furthermore, conversion of dsRNAs into 21 or 24-nt siRNAs by DCL2 or DCL4 and loading into AGO1/AGO4 results in target cleavage (Allen et al. 2004). Importantly, phasiRNAs are triggered by either "one-hit" (one binding site in the target) 


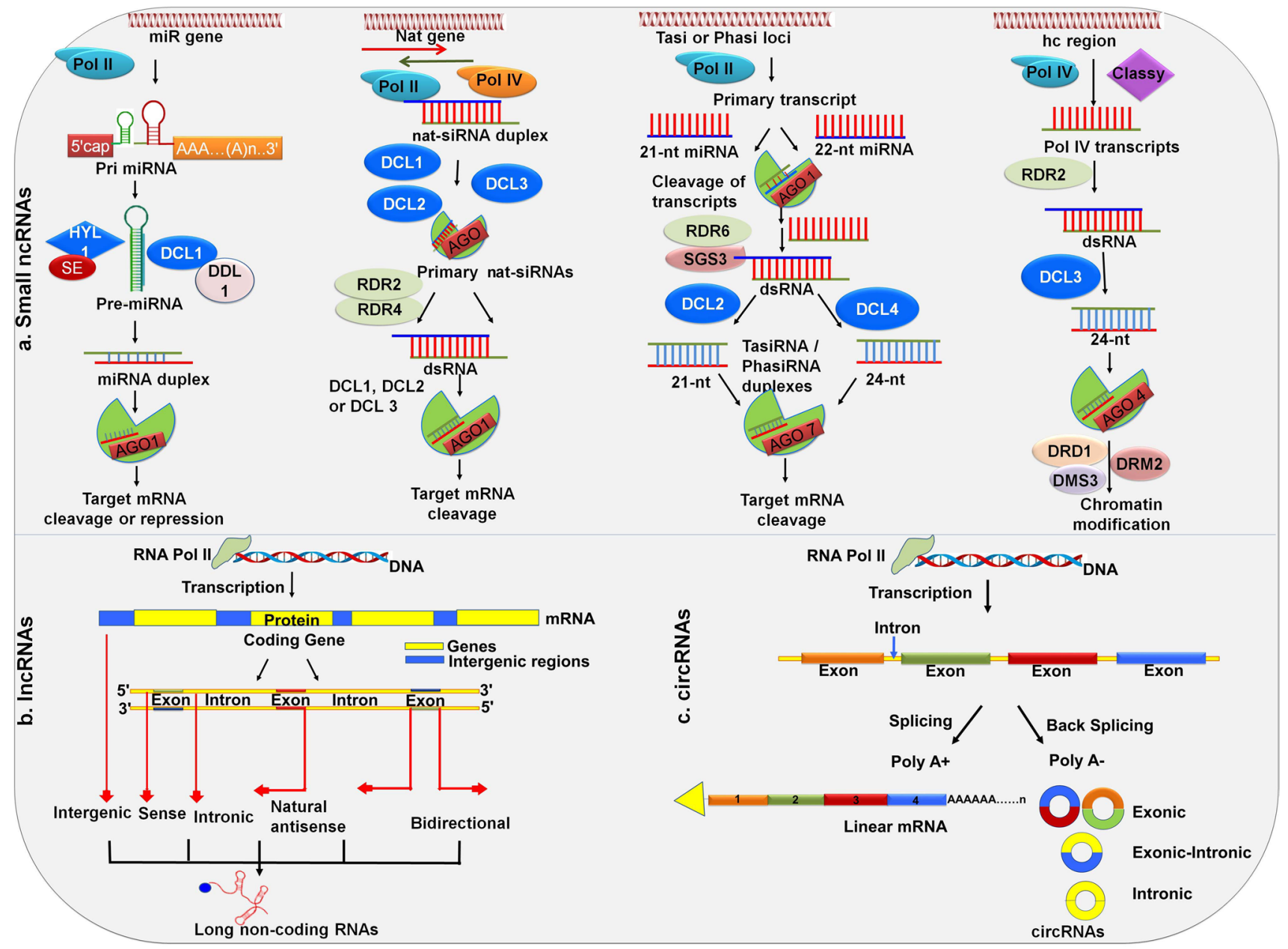

Fig. 2 Canonical biogenesis of regulatory non-coding RNAs. a Small ncRNA biogenesis (left to right): microRNAs (miRNAs) transcribed from respective miR genes through the intermediate stem-loop structures called precursor miRNAs (pre-miRNAs) and miRNA duplexes by polymerase II (pol II) and other proteins. Further mature miRNA strand is incorporated to Argonaute (AGO) I for post-transcriptional gene silencing (PTGS) by target cleavage or repression. Next to the miRNAs, a class of natural antisense RNAs (natsiRNAs or nat-siRNAs) derived from the nat genes by the action of pol II or pol IV by forming double-stranded RNAs (dsRNAs) as intermediates either from overlapping loci or from complementary loci to generate cis and trans natsiRNAs. Trans acting siRNAs (tasiRNAs or ta-siRNAs) or phasiRNAs are transcribed from respective tasi or phasi genes by pol II through the formation of dsRNAs as intermediates. Action of

or "two-hit" (two binding sites in the target) models (Fei et al. 2013). Contrarily, hcsiRNAs (23-24 nt) involve in the transcriptional gene silencing (TGS) by guiding the methylation of DNA and/or histones through the RNA-directed DNA methylation (RdDM) (Matzke et al. 2015) (Fig. 2a). These are derived from the transposable elements (TE) and repeats of hc regions by the action of Pol IV and CLASSY1 (CLSY1) followed by RDR2 and DCL3 to generate hesiRNAs, that aid in the site-specific chromatin modifications (Yu et al. 2019).
RNA-dependent RNA polymerase 6 (RDR6) on dsRNAs results in the formation of 22-nt or 24-nt tasiRNAs or phasiRNAs. natsiRNAs/ tasiRNAs/phasiRNAs are involved in target gene cleavage. Heterochromatic siRNAs (hcsiRNAs) are derived by the transcription of heterochromatin regions with Pol IV and RDR2 through the formation of intermediate dsRNAs. Further subsequent process of dsRNAs results in the formation of 24-nt hcsiRNAs that mainly involves chromatin modifications. b Long non-coding RNAs (lncRNAs): LncRNAs are transcribed by Pol II, and based on their relative position of their transcription from the genome, lncRNAs are classified in to intergenic, sense, intronic, natural antisense, and bidirectional. c Circular RNAs (circRNAs): circRNAs are derived from the exons, introns, or exonicintronic regions through back-splicing of protein-coding genes

\section{IncRNAs and circRNAs}

lncRNAs are usually derived from genomic regions that lack coding potential and possess the transcript length $>200 \mathrm{nt}$. The majority of lncRNAs that are transcribed by Pol II possess a 5' cap and non-adenylated or poly-adenylated $3^{\prime}$ tail. Other RNA polymerases like Pol IV and Pol V also play a central role in the biogenesis of IncRNAs (Wierzbicki et al. 2008, 2009). IncRNAs transcribed from Pol IV and Pol V lack poly-A tails and merely 


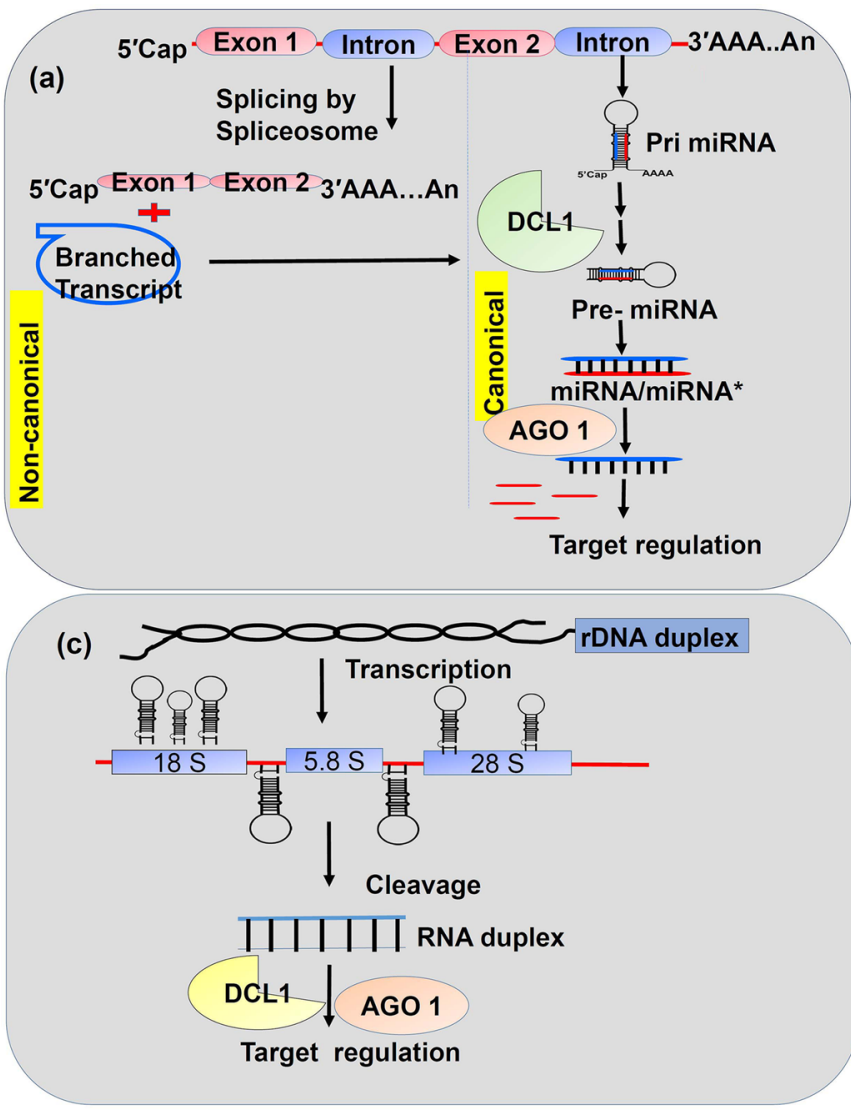

Fig. 3 Non-canonical biogenesis of non-coding RNAs. a Non-canonical biogenesis of miRNAs occurs through mirtron-like transcripts which can be spliced and debranched in to pre-miRNA hairpins that bypasses the pri-miRNA step in canonical biogenesis. Debranched mirtron-like transcripts enters canonical miRNA pathway and later cleaved by Dicer like1 (DCL1) and Argonaute 1 (AGO1) proteins. b tRNA-derived non-coding RNAs pathway includes the action of

less expressed when compared to lncRNAs derived from Pol II and play a significant role in driving RdDM (Budak et al. 2020; Zhou and Law 2015). Based on the biogenesis loci, IncRNAs are further classified as intergenic, intronic, sense or overlapping, antisense, and bidirectional (Budak et al. 2020) (Fig. 2b). lncRNAs regulate gene expression at transcriptional and post-transcriptional levels through different mechanisms. They act as scaffolds by interacting with chromatin regulatory proteins, as miRNA decoys (sponges/target mimics), and as mediators in epigenetic silencing (Wang et al. 2018a).

In addition, circRNAs, a distinct class of endogenous ncRNAs characterized by covalently closed structures without $5^{\prime}$ or $3^{\prime}$ ends, are derived through non-sequential back-end splicing from the precursor mRNAs by Pol II (Zhang et al. 2016a). These are categorized into exonic, intronic, intergenic, and exon-intronic, based on the derived genomic region and regulates the gene expression by acting as sponges for miRNAs (Sablok et al. 2016) (Fig. 2c). (b)

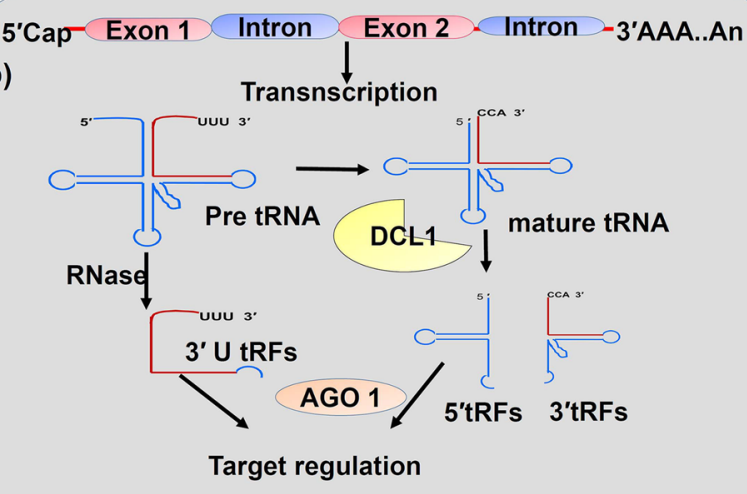

(d)

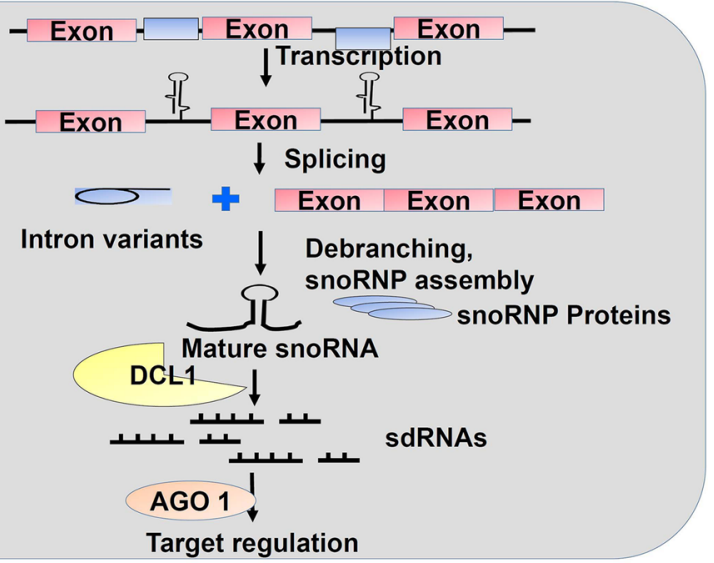

RNases on both precursor tRNAs (pre tRNA) and mature tRNAs that give rise to small RNAs like 3' U tRFs, 5' tRFs, and 3' tRFs. c Small RNA duplexes derived from ribosomal DNA (rDNA) through transcription and subsequent cleavage by DCL1 functions like miRNAs by entering in to AGO pathway. d Small RNAs derived from snoRNAs (sdRNAs) regulate their target transcripts by entering in to DCL1 and AGO1 pathway like miRNAs

\section{Non-canonical biogenesis}

Besides canonical, ncRNAs choose alternative routes for biogenesis through dicer-independent mechanisms and were described as "non-canonical" pathways, which include few miRNAs, and derived ncRNAs. Here, (1) pre-miRNA hairpin structures called "mirtrons" are generated through splicing mechanism instead of DCL1 (Budak and Akpinar 2015; Meng and Shao 2012), 2) DLC2, DCL3, and DCL4, act on long inverted repeat transcripts which results in miRNA species varying in length, (3) processing of primiRNAs in reverse orientation from loop to the base generates multiple duplexes of miRNA/miRNA* rather than a single duplex (Fig. 3a) (Budak et al. 2015; Sobkowiak et al. 2012). Sometimes, the unusual genetic loci can also harbor non-coding small RNAs. For example, (1) the derivatives of rRNAs, tRNAs, snoRNAs, snRNAs, and transposons are derived ncRNAs (Son et al. 2013), (2) small RNAs (18-26 nt) derived from tRNA are tRNA-derived fragments (tRFs), 
processed by either DICER or by Dicer-independent pathway through the action of ribonuclease (RNase) (Fig. 3b). Based on their derived region, the tRFs are categorized into 5'-tRFs, 3'-tRFs, and 3'-U tRFs. In plants, the regulatory role of tRFs through RNA degradation and translational inhibition was studied in Arabidopsis (Zhang et al. 2009; Zhu et al. 2018), (3) miRNAs derived from ribosomal DNA (rDNA) have also been reported in humans and plants (De Paola et al. 2016; Mangrauthia et al. 2018) (Fig. 3c), (4) small RNAs derived from snoRNA (sdRNA) possess a function as like miRNAs (Taft et al. 2009) (Fig. 3d), (5) TE-derived ncRNAs are transcribed from TE genomic regions through Pol II and processed by RDR6 and DCL2/4 to form 21 or 22-nt siRNAs and thus targets TE mRNAs for degradation in association with AGO1 (Cho and Paszkowski 2017). The activation of several TEs during hypomethylation results in epigenetically activated siRNAs (easiRNAs) (Creasey et al. 2014). Furthermore, TEs can establish RNA hairpin structures and proces them through miRNA biogenesis pathways to form TE-derived miRNAs (Creasey et al. 2014; Nosaka et al. 2012). In addition, TE-derived lncRNAs have also been reported in plants (Wang et al. 2015a).

The non-canonical pathways and unconventional genetic loci of ncRNAs biosynthesis in plants indicate the unknown complexity of gene regulation. More in-depth studies in this area of research will help in understanding the precise regulation of ncRNAs.

\section{ncRNAs isoforms, mechanisms, and biological significance}

The combination of high-throughput sequencing technologies and bioinformatics advancements aided the discovery of novel regulatory small RNAs called "isomiRs," the canonical variants of miRNAs (Jeong et al. 2013). These multiple miRNA isoforms/isomiRs are usually generated from a single locus by DICER from imprecise cleavage, which is perfectly complementary to their pre-miRNA sequences. IsomiRs differ from the canonical miRNAs by nucleotide variation in their $5^{\prime}$ or $3^{\prime}$-end or both of the seed regions thus targeting a different mRNA molecule. Based on the variations in length, isomiRs are classified as $5^{\prime}, 3^{\prime}$, and polymorphic isomiRs. Comparatively, the existence of $3^{\prime}$ substituted isomiRs is more evident in plants than $5^{\prime}$ substituted isomiRs, which are considered as a potential source for target site alterations (Balyan et al. 2020; Yang et al. 2019). In plants, the first isomiRs were reported in rice followed by peach, Arabidopsis, common bean, etc., and their decisive role in plant development and stress response also has been elucidated (Jeong et al. 2013; Yang et al. 2019). IsomiRs regulates post-transcriptional responses by acting as canonical partners to miRNAs. These isoforms proved to be functionally capable of target cleavage and thereby involved in the miRNA regulatory network. Studies suggested that canonical miRNA variants and their targets are evolutionarily conserved and are lineage-specific. For instance, miR156 is one of the broad and highly conserved miRNA family domains, and its regulation has been widely documented in plants that comprise different isoforms (Yang et al. 2019). Studying various isoforms of different miRNAs and lineage-specific isomiRs with respect to their parent miRNAs would help to understand their similar or differential roles in development, and stress responses (Budak et al. 2015; Yang et al. 2019).

\section{Regulation of ncRNAs synthesis and decay}

Besides the synthesis, the decay of the ncRNAs is equally an essential process to maintain homeostasis. Moreover, this additional layer of modulating ncRNA expression, processing, and action will provide plasticity to the roles played by ncRNAs. Regulation of production and decay of different ncRNAs is still not completely understood in plants except for miRNAs with a few examples. In Arabidopsis, cyclindependent kinase F: 1 (CDKF: 1) controls the transcription of MIR genes by regulating Pol II activity by phosphorylation. Similarly, a conserved transcriptional co-activator (a multi-subunit complex) reduces the loading of Pol II at MIR gene promoters (Hajheidari et al. 2012). Negative on TATA less2B (NOT2b) protein interacts with the Pol II for the efficient transcription of MIR genes (Wang et al. 2019a). Similarly, the cell division cycle 5 (CDC5) protein functions as a positive regulator of transcription in association with both Pol II and MIR promoters (Wang et al. 2019a). Apart from the transcription process, subsequent steps of biogenesis were also regulated. SE, dsRNA-binding protein, HYL1, and TOUGH (TGH) proteins interact with DCL1, to regulate miRNA accumulation. Similarly, feedback regulation of miRNAs biogenesis is a well-known phenomenon, where the miRNAs regulate their own biogenesis. miR162 and miR168 are the two key feedback regulatory miRNAs by targeting DCL1 and AGO1 mRNAs (Song et al. 2019; Wang et al. 2019a). Furthermore, methylation plays a prominent role in stabilizing and destabilizing the miRNAs. Usually, unmethylated miRNAs can be easily degraded either by uridylationdependent or by independent mechanisms. Methylated miRNAs can also be degraded through an unknown mechanism followed by the uridylation process. Overexpression of HUA ENHANCER1 (HEN1) SUPPRESSOR1 (HESO1) reduces miRNA accumulation in henl mutants of Arabidopsis (Song et al. 2019). Small RNA degrading nuclease (SDN) also plays an important role in the turnover of mature miRNAs, and its deactivation results in the accumulation of miRNAs and diminished plant development (Xie et al. 2015). More 
extensive studies are required to understand the network of proteins/enzymes involved in the regulation of synthesis and decay of miRNAs and other ncRNAs.

\section{Role of regulatory ncRNAs in plant growth and development}

The crucial role of regulatory ncRNAs in plant growth and development has been elucidated in detail in many reviews and research articles. Especially, there are many studies on miRNAs and lncRNAs describing their regulatory role in plant growth and development (Li and Zhang 2016; Swarup and Denyer 2018; Yu et al. 2019). Here, we primarily discussed how these ncRNAs regulate different complex networks. Different phase transitions from seed germination-vegetative-reproductive stages are crucial in the plant development process besides the growth of different tissues and organs.

The role of miRNAs as gene regulators in plant growth and development has been demonstrated in several studies (Li and Zhang 2016; Swarup and Denyer 2018; Yu et al. 2019). Majorly, plant miRNAs target different transcription factors (TFs) and genes of various pathways to regulate diverse biological processes (Fig. 4). Efforts to understand the regulatory mechanisms in controlling different plant developmental stages have led to the discovery of numerous miRNAs and their complex gene networks (Das et al. 2015; Swarup and Denyer 2018). Different miRNA-target modules especially, miR156-SQUAMOSA PROMOTER BINDING PROTEIN-LIKE (SPL), miR159-MYELOBLASTOSIS (MYB), and miR172-APETALA 2 (AP2) are the key regulators in different plant developmental phase transitions (Ma et al. 2020; Swarup and Denyer 2018). These modules act either as positive or as negative regulators in promoting from one phase to another. For instance, in Arabidospsis, miR156$S P L$ module acts as a negative regulator for germination-vegetative-reproductive stage transitions, where the decreased levels of miR156 elicit the $S P L$ expression to accelerate the transitions. On the other hand, miR172-AP2 module acts as a positive regulator for the same transition, where the increased levels of miR 172 decrease the AP2 expression and thus promotes transition. This clearly implicates that miRNAs can turn on and off the specific pathways by finetuning the expression of targets. Several studies showed that miRNAs establish the regulatory networks by coordinating with different hormones like gibberellic acid (GA) and abscisic acid (ABA) to control germination and dormancy processes in plants (Das et al. 2015; Liu and El-Kassaby 2017; Martin et al. 2010). For instance, miR159 plays a vital role in controlling seed dormancy and germination via altering the balance between ABA and GA hormones (Martin et al. 2010). miR159 regulates the expression of MYB TFs,
MYB33, and MYB101 to establish a positive regulation through ABA signaling in seed germination and dormancy (Reyes and Chua 2007). Furthermore, multiple members of the same gene family targeted by discrete miRNAs result in diverse biological functions. For instance, miR160 controls seed germination by negatively regulating the expression of AUXIN RESPONSE FACTORS (ARFs) in rice, and Arabidopsis (Das et al. 2015). Similarly, miR167 controls the root development by modulating the expression of $A R F 6$, and $A R F 8$ by positive regulation (Gleeson et al. 2014). In leaf, miR165/166 regulates polarity with miR390 by targeting several $A R F$ genes through the production of siRNAs (Chitwood and Timmermans 2010). These findings suggest the discrete regulatory role of miRNAs in different developmental transitions by mediating definite signaling pathways. In addition, miRNAs also act in an integrative mode on a single biological function as discussed by Yu et al. (2019). Furthermore, isoforms of a miRNA family might participate in similar biological functions either through the same or through different target genes (Alptekin et al. 2017). For instance, miR159a.1-MYB and miR159a-p5-TETRAKETIDE ALPHA-PYRONE REDUCTASE 1 (TKPRI) modules associated with male meiosis and were significantly expressed in pollen and embryo sac. Thus, the complex regulatory network of miRNA-target modules forms the molecular basis of growth and development.

Besides miRNAs, studies showed the role of siRNAs in plant development (Khraiwesh et al. 2012). The phased TAS3-tasiRNAs derived from miR390-AGO7 complex targets $A R F$ family members, which regulate various biological processes. The TAS3-tasiRNAs- $A R F$ regulatory network emerged as the most conserved module in plants by regulating developmental transitions, embryo development, root structure, shoot apical meristem (SAM) development, leaf morphology, and flower and phytohormone cross-talk (Deng et al. 2018; Xia et al. 2017; Yu et al. 2019). In Arabidopsis, TAS4-tasiRNAs are triggered by miR 828 targeted $M Y B$ genes (PRODUCTION OF ANTHOCYANIN PIGMENT 1 (PAP1), PAP2, and MYB113), which are involved in the regulation of anthocyanin biosynthesis pathway (Zhou et al. 2020). Two homologous $M Y B$ genes regulate fiber development in cotton, and interestingly, these regions were TAS4 orthologs in Arabidopsis and cotton, where one of the MYB genes is targeted by miR 828 to generate 21 -nt phasiRNAs. Furthermore, miR828 derived cis, trans siRNAs and phasiRNAs also regulate trichome development (Guan et al. 2014). Besides these studies, the role of natsiRNAs and hcsiRNAs in plant development is comparatively less. The involvement of cell-specific natsiRNA in the double fertilization process by regulating KOKOPELLI (KPL) and ARIADNE14 (ARI14) genes has been studied in Arabidopsis (Borges and Martienssen 2015). 


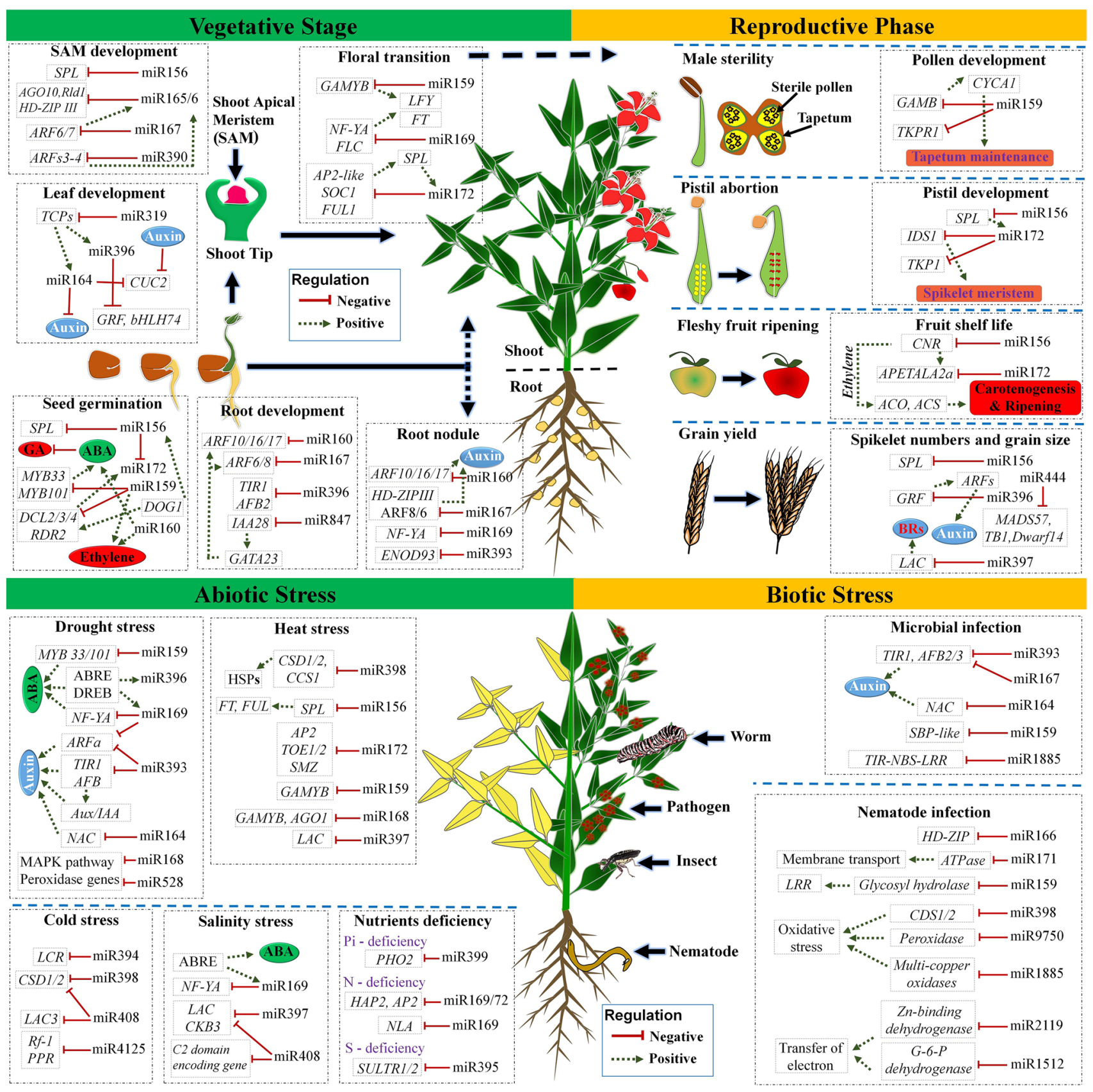

Fig. 4 Regulation of miRNAs in plant development and stress mechanisms. Different miRNA-target modules involved in plant growth, development, abiotic and biotic stress episodes, and their regulation

In addition to the sncRNAs, IncRNAs tend to play a significant role in different developmental processes of plants by regulating the expression of neighboring genes by acting in cis and distant genes by trans modes (Yu et al. 2019). The possible mechanisms of the regulation are by chromatin/ histone modifications through recruiting proteins, by acting as miRNA mimics, transcriptional regulation, and silencing or post-translational modifications. For instance, lncRNAs acting in cis were identified in root growth regulation and flowering time of Arabidopsis. The expression of PINOID $(P I D)$, an auxin transport gene, is regulated by the long intergenic non-coding RNA (lincRNA) AUXIN-REGULATED PROMOTER LOOP (APOLO) through the formation of chromatin loop, thus regulating the root growth in Arabidopsis (Ariel et al. 2014). Similarly, the lncRNA, COLD-INDUCED LONG ANTISENSE INTERGENIC NONCODING RNA (COOLAIR) regulates the expression of FLOWERING LOCUS C (FLC) gene through the association 
of chromatin and recruiting chromatin modifiers, and thus regulating flowering time and seed dormancy (Chen and Penfield 2018). Another example of cis acting lncRNA was LAIR (L-(LEUCINE-RICH REPEAT RECEPTOR KINASE (LRK)) ANTISENSE INTERGENIC RNA), which regulates grain yield in rice by recruiting chromatin-modifying complexes to increase H3K4me3 and H4K16ac levels of its target $L R K$ gene (Wang et al. 2018a). Similarly, few examples of trans acting lncRNAs were ALTERNATIVE SPLICING COMPETITOR (ASCO), which regulates the NUCLEAR SPECKLE RNA-BINDING (NSR) mRNA by modulating the alternative splicing patterns during root development of Arabidopsis (Bardou et al. 2014). Also, another lincRNA, HIDDEN TREASURE 1 (HIDI) promotes photomorphogenesis and represses the greenness of cotyledons by regulating the expression of PHYTOCHROME-INTERACTING FACTOR 3 (PIF3) through chromatin interaction (Wang et al. 2014). Furthermore, increased expression of lncRNA, long-day-specific male-fertility-associated RNA (LDMAR), is essential for the pollen development during long-day conditions and single-nucleotide polymorphism (SNP) at the LDMAR locus increases RdDM at its promoter region and reduces LDMAR transcription (Ding et al. 2012).

Furthermore, studies on understanding the role of circRNAs in plant development have also been documented (Chu et al. 2018; Liu et al. 2017a; Zhang et al. 2020; Zhao et al. 2019). In Arabidopsis, increased expression of circRNAs associated with porphyrin, chlorophyll metabolism, and signal transduction of hormones was detected during leaf senescence (Liu et al. 2017a). In another study, a circRNA derived from the 6th exon of SEPALLATA3 (SEP3) negatively regulates its own gene by acting in $c i s$ by binding to its cognate DNA locus and forming R-loop. This results in transcriptional pausing and increased abundance of alternative splice variants of the parental transcript (SEP3), which in turn results in the surge of floral homeotic phenotypes (Conn et al. 2017). The overexpression of rice circRNA, Os08circ 16564, resulted in a severe decline of the $A K 064900$ gene, which has been involved in the development of rice spikelet and floral organs (Lu et al. 2015). Though sequencing technologies aid the identification of several circRNAs in plants, the regulation of circRNAs still needs more indepth studies.

In addition, discoveries of other ncRNAs have added a piece of interesting information in this area of research (Cho 2018; Cho and Paszkowski 2017; Martinez et al. 2017). The tRNA-derived ncRNA, tRFGlu-CTC-5A, showed specific expression in flower tissues, while 5'-tRFs were accumulated in pollen tissue of Arabidopsis (Martinez et al. 2017). Expression of TE-derived lncRNA called MIKKI was detected in rice roots, and it has multiple intron sites that produce a binding site for miR171 upon splicing (Cho and Paszkowski 2017). Despite appreciable success in understanding the role of miRNAs in plant development, the functions and biological mechanisms of emerging ncRNAs like circRNAs and derived ncRNAs are still unclear. Intensive efforts are needed to ascertain the functional and regulatory role of emerging ncRNAs in concert to coordinate the different biological functions and mechanisms during plant development.

\section{Role of regulatory ncRNAs in plant stress responses}

Regulatory roles of ncRNAs in various stress episodes also have been well-studied in plants. Activation of different regulatory ncRNAs by biotic and abiotic stress elicitors leads to the regulation of crucial stress-responsive pathways through target transcripts (Fig. 5). As stated in the earlier section, among different ncRNAs, miRNAs are the extensively investigated class followed by siRNAs and lncRNAs (Khraiwesh et al. 2012; Song et al. 2019; Yu et al. 2019). Regulation of gene expression mediated by miRNAs during different stress responses (drought, heat, salinity, cold, nutrient, and pathogen) has been exemplified in a different model and crop plants such as Arabidopsis, wheat, rice, maize, and barley (Barciszewska-Pacak et al. 2015; Ferdous et al. 2017; He et al. 2019; Hua et al. 2019; Mangrauthia et al. 2017a; Sailaja et al. 2014). In addition, there are comprehensive reviews that delineated the expression and regulation of different conserved miRNAs during various environmental stress episodes (Ferdous et al. 2015; Megha et al. 2018; Song et al. 2019; Zhao et al. 2016).

The wide range of miRNAs expression in each stress response has been witnessed in many plant species. However, few miRNA-target modules can show definite expression patterns against specific stress by regulating target genes, and their pattern of expression can be conserved across different plant species (Song et al. 2019). In addition to plant growth and development, the role of conserved miRNAtarget modules is also crucial in conferring stress tolerance by integrating with metabolic pathways. Well-known conserved miRNA-target modules such as miR156-SPL, miR159-MYB, miR160-ARF, miR164-NAC (NAM, ATAF, and $C U C$ ), miR167-ARF, miR169-NUCLEAR TRANSCRIPTION FACTOR-Y (NFY), miR319-TEOSINTE BRANCHED/ CYCLOIDEA/PROLIFERATING CELL FACTORS (PCF) (TCP), miR394-LEAF CURLING RESPONSIVENESS (LCR), miR396-GROWTH REGULATING FACTOR (GRF), and miR398- COPPER/ZINC SUPEROXIDE DISMUTASE $(C S D)$ are known to play an important regulatory role in different stress environments to mitigate the detrimental effects (Fig. 4). For instance, different miRNAs are known to target TFs in phytohormone regulation, such as ABA, GA, ethylene signaling, and auxin signaling under drought conditions 


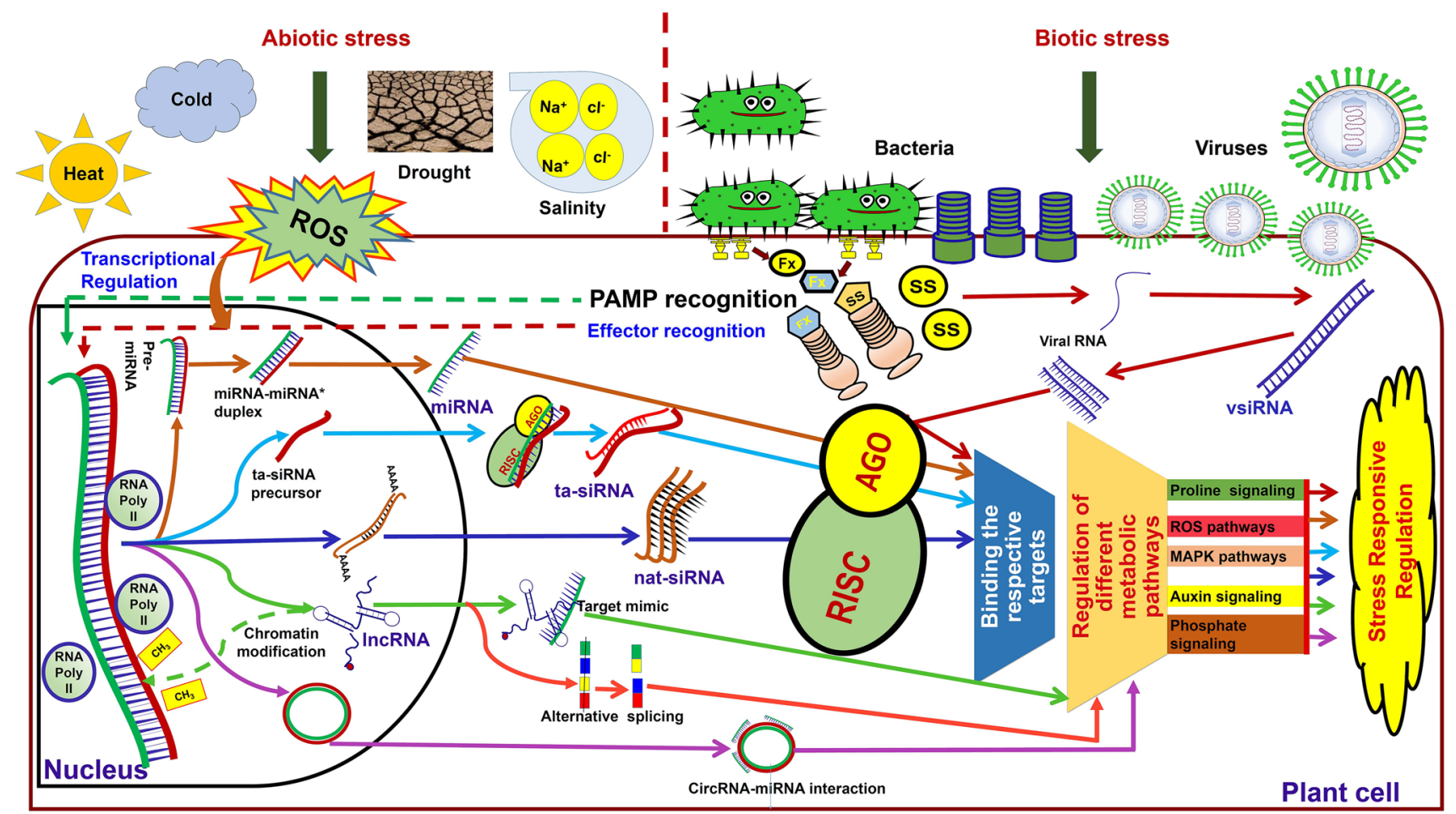

Fig. 5 General model of stress-responsive regulation by regulatory non-coding RNAs in abiotic and biotic stresses. Abiotic and biotic stresses elicit the production of reactive oxygen species (ROS) and pattern-associated molecular pattern (PAMP) recognition in plants through signal perception. Synthesis of different classes of non-coding RNAs (ncRNAs) in response to ROS and PAMP is one of the defensive mechanisms in plants. miRNAs and other small ncRNAs thus produced in response to stress, bind to their respective target genes with the aid of Argonaute (AGO) proteins by forming RNAinduced silencing complex (RISC), and thus regulate the expression

(Ferdous et al. 2017). The miR167-ARF module regulates the auxin signaling pathway during drought stress. ARF6 and $A R F 8$, the targets of miR 167 , negatively regulates auxin signaling pathway through GRETCHEN HAGEN 3 (GH3). During drought, miR167 was upregulated in Arabidopsis, wheat, and maize, while it was downregulated in rice (Song et al. 2019). Similarly, miR169-NFY module also plays a significant role during water-deficit conditions. In Arabidopsis, tomato, and Medicago, downregulation of miR169 enhances the expression of its target, $N F-Y$ ( $\mathrm{Li}$ et al. 2008; Megha et al. 2018; Zhang et al. 2011a). The increased expression of NF-Y in stomatal guard cells enhances the drought tolerance by controlling the aperture of the guard cell in plants (Li et al. 2008). Besides, miR160-ARF, miR156$S P L$, miR159-MYB33, miR164-NAC, miR172-AP2 etc. modules are also shown to be involved in the regulation of drought stress response (Song et al. 2019). Similarly, several miRNAs were also identified in the regulation of plant's heat stress response (Mangrauthia et al. 2017a; Ravichandran et al. 2019; Sailaja et al. 2014; Wang et al. 2011). In of target genes. Long non-coding RNAs (IncRNAs) regulate the gene expression by mimicking the miRNA targets or through alternative splicing or by chromatin modification. Circular RNAs (circRNAs) regulate the gene expression by acting as miRNA sponges. Successively, thus generated ncRNAs regulate the different metabolic pathways that primes in the stress-responsive regulatory mechanism. BEbacterial effector, SS- silencing suppressor, dsRNA- double-stranded RNA, PRRs- pathogen recognition receptors, R proteins- resistance protein, RISC- RNA-induced silencing complex

Arabidopsis, Brassica, and Populus, one of the important and most conserved miRNA-target modules as a part of the heat stress response is miR398-CSD (Guan et al. 2013; Yu et al. 2012). In Arabidopsis, increased expression of miR398 enhanced the heat tolerance in plants by negatively regulating the expression of its targets - CSD1, CSD2, and COPPER CHAPERONE OF CSD (CCD) (Guan et al. 2013; Lu et al. 2013). Decreased levels of $C S D 1, C S D 2$, and $C C D$ aids in the accumulation of heat shock transcription factors (HSFs) and heat shock proteins (HSPs). Furthermore, other conserved modules, viz., miR156-SPL, miR172-AP2 also contribute to heat stress response in plants (Song et al. 2019; Zhao et al. 2016). In addition, the highly conserved miR394-LCR module participates in the cold stress response of plants. In Arabidopsis, overexpressed miR394a plants exhibit cold tolerance by negatively regulating the $L C R$ gene (Song et al. 2016). Furthermore, the increased expression of genes encoding C-REPEAT BINDING FACTORS ( $C B F s$ ) or DEHYDRATION-RESPONSIVE ELEMENT-BINDING FACTORS 1 (DREB1) in overexpressed miR394 and lcr 
mutant plants exhibits cold stress tolerance, which infers the positive regulation of miR394 through CBF-dependent pathway in acquiring cold stress tolerance (Song et al. 2016). Furthermore, the regulatory role of conserved miR319-TCP module conferring salinity tolerance in plants evidenced through overexpression studies of osa-miR319a in bentgrass (Agrostis stolonifera) (Zhou et al. 2013). In addition to the abovementioned studies, the involvement of miRNAs in nutrient uptake and nutrient homeostasis also has been shown. For instance, participation of miR399-PHOSPHATE OVER ACCUMULATOR 2 (PHO2) module during phosphate deficiency, miR827-NITROGEN LIMITATION ADAPTATION (NLA) and miR169-NF-Y modules in nitrogen deficiency, and miR395-SULFATE TRANSPORTER2;1 (SULTR2) in sulfur assimilation and transportation were also studied (Song et al. 2019). Besides miRNAs, studies also suggested the role of different isomiRs in plant stress responses. For instance, the differential expression of various isomiRs of the conserved miR 156 family was identified during drought stress in maize (miR156a, b, c, d, e, h, i, and 1) and rice (miR156d-5p.2, miR156f-5p.2, miR156h-5p.2, and miR156j-5p.2) (Balyan et al. 2020; Zheng et al. 2019a). Also, during heat stress, the highly differential expression of miR156 isoform than its canonical miRNA has been witnessed in Arabidopsis, which elucidates the important regulatory role of isomiRs (Baev et al. 2014).

Furthermore, the regulatory role of miRNA-target modules during biotic stresses caused by bacteria, fungi, viruses, and insects has also been established (Brant and Budak 2018; Khraiwesh et al. 2012; Song et al. 2019) (Fig. 4). In Arabidopsis, the regulatory role of miR393-TRANSPORT INHIBITOR RESPONSEI(TIRI), AUXIN SIGNALING $F-B O X 1$ ( $A F B 2$ ), and $A F B 3$ was the first identified module as a defensive response against Pseudomonas syringae pv. tomato DC3000, a bacterial pathogen. Here, increased miR393 expression levels due to bacterial PATHOGENASSOCIATED MOLECULAR PATTERNS (PAMP) flagellin (flg22) downregulate $T I R 1, A F B 2$, and $A F B 3$, which results in increased bacterial resistance. Similarly, pathogen-associated triggered immunity in response to fungal pathogens, miR773-METHYLTRANSFERASE 2 (MET2) module, displayed enhanced resistance (Salvador-Guirao et al. 2018). Also, in rice, the miR528-ASCORBATE OXI$D A S E(A O)$ module contributes towards the enhancement of viral defense by accumulating reactive oxygen species (ROS). Upon the rice stripe virus (RSV) infection, miR528 masked by AGO 18 leads to elevated $A O$ activity and in turn helps in the accumulation of basal reactive oxygen species (ROS) to enhance antiviral defense. In addition to the above discussed prominent regulatory roles of miRNA modules in both abiotic and biotic stress responses, there are many other modules (reviewed in Song et al. 2019) and are not further discussed here.
In addition, other sncRNAs like tasiRNAs are also shown to be involved in plant stress responses. For instance, $H E A T$ INDUCED TAS1 TARGET 1 (HTT 1) and HTT 2 mRNA targets of TAS1 (trans-acting siRNA precursor 1)-derived tasiRNAs form miR173 contribute to thermotolerance in Arabidopsis ( $\mathrm{Li}$ et al. 2014a). Plants with elevated levels of TAS1-siRNAs and decreased levels of the HTT genes are sensitive to heat stress, while the plants overexpressing HTT1 and HTT2 genes exhibited enhanced thermotolerance (Li et al. 2014a). Furthermore, during phosphate homeostasis, positive regulation of protein derived from $P H O S$ PHATE1;2 $\left(\right.$ PHOl;2) gene and its cis-NAT (cis-NAT $\left.{ }_{P H O l ; 2}\right)$ in Arabidopsis has been confirmed. Downregulation of $\mathrm{cis}$ $\mathrm{NAT}_{P H O 1 ; 2}$ through RNAi revealed the impaired allocation of phosphate from root to shoot, which ultimately led to reduced seed yield by reduction of PHO1;2 proteins (Jabnoune et al. 2013). Similarly, the regulatory role of natsiRNAs during salt stress was demonstrated in Arabidopsis. natsiRNA (24 nt) generated from SIMILAR-TO-RCD-ONE 5 (SRO5) mRNA, targets D1-PYRROLINE-5-CARBOXYLATE DEHYDROGENASE (P5CDH) results in the subsequent formation of $21 \mathrm{nt}$ natsiRNAs. The generated natsiRNAs further participates in the cleavage of $P 5 C D H$ mRNA. During salt stress, induction of SRO5 protein results in the declined expression of $P 5 C D H$ activity leading to proline and reactive oxygen species (ROS) accumulation. Thus, the role of natsiRNAs of $S R O 5$ on $P 5 C D H$ genes, together with their respective proteins in osmoprotection and oxidative stress during salt stress has been confirmed (Borsani et al. 2005; Khraiwesh et al. 2012). Similarly, the role of phasiRNAs derived from miR482, miR828, and miR6455 during drought stress was studied in populus, where populus-specific miR6455 derived 22-nt phasiRNA targeted $N A C$ genes, that are known to play a crucial role in drought stress (Shuai et al. 2016). Furthermore, during biotic stress, the first plant-endogenous siRNA nat-siRNAATGB2 regulates $R$-gene-mediated ETI (effector-triggered immunity) towards bacterial pathogen Pseudomonas syringae (Ps) infection (Navarro et al. 2006). Induction of this siRNA inhibits the expression of antisense target PENTATRICOPEPTIDE REPEAT PROTEIN-LIKE (PPRL), a negative regulator of $R P S 2$-mediated ETI in response to $P S$. Generated endogenous siRNA, nat-siRNAATGB2, aids in $R$-gene, $R P S 2$-mediated race-specific disease resistance by inhibiting the expression of predicted negative regulator PPRL gene (Katiyar-Agarwal and Jin 2010). Furthermore, in Arabidopsis, phasiRNAs derived from $P P R$ genes confers a defensive response against the Phytophthora capsici infection (Hou et al. 2019). In tomato, transgenic lines expressing short tandem target mimic (STTM) RNAs of miR482/2118 confirm the role of derived phasiRNAs in the regulation of nucleotide-binding site leucine-rich repeat (NLR) genes and the important role of NLR proteins in conferring disease 
resistance against bacterial and oomycete pathogens (CantoPastor et al. 2019). Similarly, overexpression of two tasiRNAs derived from TAS1 and TAS2 loci resulted in reduced virulence against the fungal pathogen Botrytis cinerea (Cai et al. 2018). Also, a study by Wu et al. (2020) reported the crucial role of 22-nt siRNAs derived from nitrate reductase (NIA1 and NIA2) genes helps in plant adaptation to different environmental stress responses by inducing gene silencing and translational repression. In addition to the mechanistic theme of regulation by sncRNAs, the emerging lncRNAs also have considerable attention for their regulatory role in plant stress responses.

lncRNAs that are responsive to different abiotic and biotic stresses also have been identified in different plant species. For instance, drought-responsive lncRNAs have been identified in Arabidopsis, populus, maize, rice, etc., (Chung et al. 2016; Di et al. 2014; Pang et al. 2019; Qin et al. 2017; Shuai et al. 2014). During stress periods, it is evident that lncRNAs regulate the expression of multiple genes through possible mechanisms and act as potential gene regulators in different plant biological processes. For instance, in Arabidopsis, the IncRNA, DROUGHT INDUCED LNCRNA (DIR) is responsive to drought and salinity stress and acts as a positive regulator by modifying the expression of a series of genes. The overexpressed DIR plants exhibited enhanced drought and salinity tolerance (Qin et al. 2017). In rice, genes encoding for zinc-finger proteins of drought QTL region, qSDT2-1, were found to be the predicted targets of identified lncRNAs, which signifies their regulatory role in drought stress (Weidong et al. 2020). Similarly, heat stress-responsive IncRNAs were also identified in brassica, cassava, rice, etc., (Ding et al. 2019; Luo et al. 2018; Wang et al. 2019b). In Brassica rapa, two heat stress-responsive lncRNAs identified as endogenous target mimics for miR164a and contrasting expression of both miRNA and lncRNA define their important role in heat stress response (Wang et al. 2019b). Furthermore, different abiotic stress-responsive lncRNAs act as target mimics for miR156, miR159, and miR172, thus involves in the regulation of various stress-responsive genes ABA, ethylene signaling, HSPs, and HSFs pathways (Ding et al. 2019; Wang et al. 2019b). Similarly, cold and salinity-responsive lncRNAs were identified in several plant species (Karlik and Gozukirmizi 2018; Qin et al. 2017; Wang et al. 2015b, 2019c). Two lncRNAs, COOLAIR and COLD ASSISTED INTRONIC NON-CODING RNA (COLDAIR), promote flowering in plants during cold conditions (Whittaker and Dean 2017). Similarly, signatures of lncRNA regulation in biotic stress responses were evident from different studies (Nejat and Mantri 2017; Yu et al. 2019). For instance, in tomato during Phytophthora infestans infection, the lncRNA16397 induces the expression of $G L U$ TAREDOXIN 22 gene by acting in cis and resulted in the enhanced resistance (Cui et al. 2017). Collectively, these results demonstrate the complex regulatory function of lncRNAs in defensive pathways by modulating the expression of defense responsive genes.

Similarly, the research on stress-responsive circRNAs and derived ncRNAs is in the course of its way. The expression of stress-responsive circRNAs using high-throughput sequencing technologies has been identified. In wheat, Wang et al. (2016a) identified 62 circRNAs in response to dehydration stress. Similarly, in pear fruits, 23 circRNAs showed increased expression during drought stress (Wang et al. 2018b). Furthermore, the expression of circRNAs in response to bacterial pathogen infection by Pseudomonas syringae pv. actinidiae (PSA) in kiwi fruits and by Pectobacterium carotovorum subsp. Brasiliense $(P C B)$ infection in potato delineates their role in biotic stress (Wang et al. 2017a; Zhou et al. 2018). A recent study by Fan et al. (2020) in rice showed the contribution of circRNAs in response to Magnaporthe oryzae, a fungal pathogen. The high diversity of circRNAs with tolerant genotype (IR25) during $M$. oryzae infection is due to more $3^{\prime}$ and $5^{\prime}$ alternative backsplicing and complex splice sites. Furthermore, the role of circR5g05160 in enhancing immunity against $M$. oryzae has been reported (Fan et al. 2020). Besides circRNAs, accumulation of different derived ncRNAs such as tRFs (tRNA-ValCAC, tRNA-Thr-UGU, tRNA-Tyr-GUA, and tRNA-Ser-UG) has been reported during heat and osmotic stress in wheat and phosphate stress in Arabidopsis and barley (Hackenberg et al. 2013; Hsieh et al. 2010; Wang et al. 2016b). Furthermore, activation of TE-derived lncRNA11195 after various abiotic stress treatments in Arabidopsis revealed the important role of transposon-derived lncRNAs in stress responses (Wang et al. 2017b). Though sequencing technologies expedite our understanding on the circular and derived ncRNAs in plants, still their functional characterization and in-depth investigation are prerequisite to assign the exact role of these emerging regulatory non-coding RNAs. We have summarized the stress-responsive regulatory non-coding RNAs and their expression which are valuable molecular resources in Tables S1 and S2, to understand their regulatory patterns associated with stress tolerance and plant defense mechanisms.

\section{Harnessing the regulatory ncRNAs for crop improvement}

Utilizing the available ncRNAs' information and their regulation would be a desirable application to address food and nutritional security. To use the ncRNAs' information in the improvisation of key traits in various crops, different molecular genetics-based approaches have been employed. There are some classic reports which demonstrate the effect of single miRNA manipulation for diverse traits such as increased 
crop yield, biomass, and stress tolerance (Zhang and Wang 2016). For instance, overexpression of evolutionarily conserved miR156 in tomato plants showed association with fruit size (Zhang et al. 2016b). The SPL genes, targets of miR156, showed a positive association with rice yield (Jiao et al. 2010; Wang et al. 2012). In rice, miR156 regulates the expression of SPL13, SPL14, and SPL16 genes that in turn govern the regulation of grain size and panicle (Jiao et al. 2010; Tang and Chu 2017; Wang et al. 2012). Similarly, increased expression of miR397 showed a positive correlation with the grain size and yield by regulating its target gene, LACCASE (LAC) in rice (Zhang et al. 2013a). Wang et al. (2016c) reported enhanced expression of miR444 in rice, which resulted in the downregulation of MAD23, $M A D 27 a$, and MADS57 during RSV infection, through activating RDR1-dependent antiviral RNA-silencing pathway.

Further, artificial miRNA (amiRNA/amiR) has also been suggested as a potential approach for crop improvement by constructing amiRNAs to regulate the target gene expression (Rosa et al. 2018; Zhang et al. 2018a). One such example is the construction of amiR $159 \mathrm{~b}$ to target three crucial genes involved in seed oil metabolism, viz., fatty acid $\Delta^{12}$ desaturase 1 (FAD2), fatty acid elongase 1 (FAE1), and fatty acyl-ACP thioesterase B (FATB) for high oleic content in Arabidopsis (Belide et al. 2012). The ability of amiR to target multiple traits is an added advantage to this approach. For instance, Ai et al. (2011) showed that co-expression of various amiRNAs targeting different viruses in transgenic plants leads to multiple virus resistance (Ai et al. 2011). amiRNAs designed from miR159a, miR167b, and miR171a precursors of Arabidopsis targeting expression of suppressor HC-PRO and P25 confer resistance towards Potato virus $Y$ $(P V Y)$ and Potato virus $X(P V X)$, respectively.

From markers' perspective, single-nucleotide polymorphisms (SNPs) are present abundantly in ncRNA regions which is an essential feature in crop improvement as prospective biomarkers (Fabbri et al. 2019). The base composition for miRNA/premiR sequence is very crucial for its function and the altered bases/SNPs in the premiR sequence resulted in the unstable secondary structure. Some of the important agronomic traits like grain length and seed type differentiation were found to be associated with SNPs in premiR of miR2923a (Wang et al. 2013). These variations in ncRNAs can be further exploited to improve agronomic traits of interest. Similarly, the PHOTOPERIOD-SENSITIVE GENIC MALE STERILITY 1 (Pmsl) locus encodes lncRNA, PMSIT, specifically expressed in young panicles of rice (Fan et al. 2016). miR2118 targets PMS1T to produce 21-nt phasiRNAs that specifically accumulated in the male sterile line during long-day conditions. SNP in PMS1T nearby miR2118 recognition site suggest its possible mechanism in reproductive development of rice. Availability of whole genome sequences, followed by resequencing of multiple lines for a given crop species, provides an opportunity to look into the natural variations existing in the genome. Combining such information with the transcriptomic studies may aid in the identification of the functional role of such variations mediated through ncRNAs.

In addition, studies showed that genome editing with site-specific nucleases, especially with type II clustered regularly interspaced short palindromic repeats (CRISPR)/ CRISPR-associated protein 9 (Cas9) system, is the powerful genome editing approach for functional studies in plants (Basso et al. 2019; Chen et al. 2019; Mangrauthia et al. 2017b). CRISPR/Cas9 has shown the possibility to overcome the limitations associated with RNAi by contributing to complete gene knockout and reduced off-target activities (Basso et al. 2019). Localization of certain ncRNAs in the nucleus possesses challenges with the implementation of RNAi as it is limited to the cytoplasm, where RISC is located. In the case of plants, the application of genome editing in crop improvement by targeting ncRNAs is in the emerging stage and efforts are being made to use the system efficiently (Basso et al. 2019). For instance, Jacobs et al. (2015) targeted two miRNAs, miR1514 and miR1509, in soybean with Cas9 and demonstrated the strong potential of targeting short ncRNA as a target using CRISPR/Cas9 (Jacobs et al. 2015). In a similar way, by using the CRISPR/Cas9 non-homologous end joining (NHEJ) strategy, one can introduce indels at pre-miRNA sequences or the miRNA processing sites of $M I R$ genes to regulate miRNA biogenesis (Zhou et al. 2017). Collectively, the abovementioned precision technologies would help in the effective utilization of ncRNAs' information in crop improvement by developing cultivars with desirable characteristics.

This review on diverse ncRNAs ranging from the most familiar (miRNAs, siRNAs) to the emerging ncRNAs (lncRNAs, circRNAs, tRFs, and rDNA-derived miRNAs) and their isovariants identified in different plant species provides a better understanding of their functions at multiple stages of transcriptional and post-transcriptional gene regulation. Of note, the intervention of ncRNAs in the epigenetic mechanisms highlights their potential, leading to genotypic/phenotypic variations across the plant species. We speculate that discussed ncRNAs were having their own decisive role in governing the regulation of plant growth, development, and environmental stress responses. The basic understanding of the characteristic features and functions of heterologous ncRNA species helps to link ncRNA function to the specific plant trait. It also highlights the crucial role of various ncRNAs, including target genes and their expression profiles under biotic and abiotic stresses, which will facilitate the trait-specific ncRNA selection and its deployment in crop engineering. 


\section{Conclusions and future perspectives}

This review expands our knowledge about the intertwined regulatory role of ncRNAs. With the advent of deep sequencing technologies, the identification of diverse ncRNAs and their profiling is increasing at unprecedented levels. In this context, we attempted to articulate the classification, biogenesis, and regulatory roles of the available plant ncRNAs. Moreover, deep-diving into ncRNA biogenesis is essential, as the knowledge of proteins/enzymes which are controlling the expression/decay of ncRNA is meager. In short, it was suggested that biogenesis of these diverse ncRNAs is induced, as per the plant developmental needs and stress challenges. To overcome the challenges of food security, functional validation of key ncRNAs and their isoforms has been piloted through the overexpression or knockout studies of siRNA/miRNAs. Of note, for the accurate view of ncRNA function, a novel tool CRISPR-Cas can be exclusively applied to prevent potential off-target mutations. So far, the functional studies on ncRNAs in plants confine mostly to miRNAs in different perspectives like development and stress-responsive regulation. On the other hand, research on other ncRNAs is still at infancy, and more intensive efforts are needed to unravel the complexity and functional role of different ncRNAs, especially circRNAs and derived RNAs. Likewise, it is still astonishing to believe how plants synchronize the accumulation of these diverse ncRNAs as per their need. Furthermore, to utilize the information of ncRNAs for crop improvement, an extensive knowledge is essential to understand their functional and regulatory role in different gene regulatory networks. In addition, it is imperative to develop a trait-specific candidate ncRNA catalogue which will be targets for engineering new crop varieties. Overall, this review will be helpful to the researchers to enhance their understanding of different classes of ncRNAs and their functional link with the plant phenotype and regulation. Though many challenges are yet to be addressed, strategic implementations of ncRNA-based approaches in molecular crop breeding will further strengthen to overcome the impending food crisis.

Supplementary Information The online version contains supplementary material available at https://doi.org/10.1007/s10142-021-00787-8.

Author contribution Idea for the article: SB and HK; literature search and drafted the review SB, RK, AKP, SS, and AJ; manuscript critically revised: SB, HK, SKM, HB, and RKV.

Funding SB received a National Post-Doctoral Fellowship (PDF/2017/002371) from the Science \& Engineering Research Board (SERB) of the Department of Science \& Technology (DST), Government of India. RKV was provided with the J C Bose National Fellowship (SB/S9/Z-13/2019) from SERB of DST, Government of India and Bill \& Melinda Gates Foundation for supporting legumes research through the Tropical Legumes project. HK acknowledges the SERB of
DST, Government of India, for providing SERB Women Excellence Award (SB/WEA-01/2017). This work has been undertaken as part of CGIAR Research Program on Grain Legumes \& Dryland Cereals (CRP-GLDC). ICRISAT is a member of CGIAR Research Consortium.

\section{Declarations}

Ethics approval Not applicable.

Consent to participate All authors have their consent to participate.

Consent for publication All authors have their consent to publish this article.

Conflict of interest The authors declare no competing interests.

Open Access This article is licensed under a Creative Commons Attribution 4.0 International License, which permits use, sharing, adaptation, distribution and reproduction in any medium or format, as long as you give appropriate credit to the original author(s) and the source, provide a link to the Creative Commons licence, and indicate if changes were made. The images or other third party material in this article are included in the article's Creative Commons licence, unless indicated otherwise in a credit line to the material. If material is not included in the article's Creative Commons licence and your intended use is not permitted by statutory regulation or exceeds the permitted use, you will need to obtain permission directly from the copyright holder. To view a copy of this licence, visit http://creativecommons.org/licenses/by/4.0/.

\section{References}

Ai T, Zhang L, Gao Z, Zhu CX, Guo X (2011) Highly efficient virus resistance mediated by artificial microRNAs that target the suppressor of PVX and PVY in plants. Plant Biol (Stuttg) 13:304316. https://doi.org/10.1111/j.1438-8677.2010.00374.X

Allen E, Xie Z, Gustafson SJW, Carrington JC (2004) Evolution of microRNA genes by inverted duplication of target gene sequences in Arabidopsis thaliana. Nat genet 36:1282-1290. https://doi.org/10.1038/ng1478

Alptekin B, Langridge P, Budak H (2017) Abiotic stress miRNomes in the Triticeae. Funct Integr Genomics 17:145-170. https://doi. org/10.1007/s10142-016-0525-9

Ariel F, Jegu T, Latrasse D, Christ A, Benhamed M, Crespi M (2014) Noncoding transcription by alternative RNA polymerases dynamically regulates an auxin-driven chromatin loop. Mol Cell 55:383-396. https://doi.org/10.1016/j.molcel.2014.06.011

Baev V, Milev I, Naydenov M, Vachev T, Apostolova E, Mehterov N, Gozmanva M, Minkov G, Sablok G, Yahubyan G (2014) Insight into small RNA abundance and expression in high-and lowtemperature stress response using deep sequencing in Arabidopsis. Plant Physiol Bioch 84:105-114. https://doi.org/10.1016/j. plaphy.2014.09.007

Balyan S, Joseph SV, Jain R, Mutum RD, Raghuvanshi S (2020) Investigation into the miRNA/5'isomiRNAs function and droughtmediated miRNA processing in rice. Funct Integr Genomics 20:509-522. https://doi.org/10.1007/s10142-020-00731-2

Barciszewska-Pacak M, Milanowska K, Knop K, Bielewicz D, Nuc P, Plewka P, Pacak AM, Vazquez F, Karlowski W, Jarmolowski A, Szweykowska-Kulinska Z (2015) Arabidopsis microRNA expression regulation in a wide range of abiotic stress responses. Front Plant Sci 6:410. https://doi.org/10.3389/fpls.2015.00410 
Bardou F, Ariel F, Simpson CG, Romero-Barrios N, Laporte P, Balzergue S, Brown JW, Crespi M (2014) Long noncoding RNA modulates alternative splicing regulators in Arabidopsis. Dev Cell 30:166-176. https://doi.org/10.1016/j.devcel.2014.06.017

Basso MF, Ferreira PCG, Kobayashi AK, Harmon FG, Nepomuceno AL, Molinari HB, Grossi-de-Sa MF (2019) MicroRNAs and new biotechnological tools for its modulation and improving stress tolerance in plants. Plant Biotechnol J 17:1482-1500. https:// doi.org/10.1111/pbi.13116

Belide S, Petrie JR, Shrestha P, Singh SP (2012) Modification of seed oil composition in Arabidopsis by artificial microRNA-mediated gene silencing. Front Plant Sci 3:168. https://doi.org/10.3389/ fpls.2012.00168

Borges F, Martienssen RA (2015) The expanding world of small RNAs in plants. Nat Rev Mol Cell Biol 16:727-741. https://doi.org/10. $1038 /$ nrm 4085

Borsani O, Zhu J, Verslues PE, Sunkar R, Zhu JK (2005) Endogenous siRNAs derived from a pair of natural cis-antisense transcripts regulate salt tolerance in Arabidopsis. Cell 123:1279-1291. https://doi.org/10.1016/j.cell.2005.11.035

Brant EJ, Budak H (2018) Plant small non-coding RNAs and their roles in biotic stresses. Front Plant Sci 9:1038. https://doi.org/ 10.3389/fpls.2018.01038

Budak H, Akpinar BA (2015) Plant miRNAs: biogenesis, organization and origins. Funct Integr Genomics 15:523-531. https://doi.org/ 10.1007/s10142-015-0451-2

Budak H, Kantar M, Bulut R, Akpinar BA (2015) Stress responsive miRNAs and isomiRs in cereals. Plant Sci 235:1-13. https://doi. org/10.1016/j.plantsci.2015.02.008

Budak H, Kaya SB, Cagirici HB (2020) Long non-coding RNA in plants in the era of reference sequences. Front Plant Sci 11:276. https://doi.org/10.3389/fpls.2020.00276

Cai Q, Qiao L, Wang M, He B, Lin FM, Palmquist J, Huang HD, Jin H (2018) Plants send small RNAs in extracellular vesicles to fungal pathogen to silence virulence genes. Science 360:1126-1129. https://doi.org/10.1073/pnas.1814380116

Canto-Pastor A, Santos BA, Valli AA, Summers W, Schornack S, Baulcombe DC (2019) Enhanced resistance to bacterial and oomycete pathogens by short tandem target mimic RNAs in tomato. Proc Natl Acad Sci USA 116:2755-2760. https://doi.org/10.1073/ pnas. 1814380116

Chen K, Wang Y, Zhang R, Zhang H, Gao C (2019) CRISPR/Cas genome editing and precision plant breeding in agriculture. Annu Rev Plant Biol 70:667-697. https://doi.org/10.1146/annurevarplant-050718-100049

Chen M, Penfield S (2018) Feedback regulation of COOLAIR expression controls seed dormancy and flowering time. Science 360:1014-1017. https://doi.org/10.1126/science.aar7361

Chen X (2009) Small RNAs and their roles in plant development. Annu Rev Cell Dev Biol 25:21-44. https://doi.org/10.1146/annurev. cellbio.042308.113417

Chitwood DH, Timmermans MCP (2010) Small RNAs are on the move. Nature 467:415-419. https://doi.org/10.1038/nature09351

Cho J (2018) Transposon-derived non-coding RNAs and their function in plants. Front Plant Sci 9:600. https://doi.org/10.3389/fpls. 2018.00600

Cho J, Paszkowski J (2017) Regulation of rice root development by a retrotransposon acting as a microRNA sponge. Elife 6:e30038. https://doi.org/10.7554/eLife.30038.001

Chu Q, Shen E, Ye CY, Fan L, Zhu QH (2018) Emerging roles of plant circular RNAs. J Plant Cell Dev 1:1-14

Chung PJ, Jung H, Jeong D, Ha SH, Do Choi Y, Kim JK (2016) Transcriptome profiling of drought responsive non-coding RNAs and their target genes in rice. BMC Genomics 17:563. https://doi.org/ 10.1186/s12864-016-2997-3
Conn VM, Hugouvieux V, Nayak A, Conos SA, Capovilla G, Cildir G, Jourdain A, Tergaonkar V, Schmid M, Zubieta C, Conn SJ (2017) circRNA from SEPALLATA3 regulates splicing of its cognate mRNA through R-loop formation. Nat Plants 3:17053. https:// doi.org/10.1038/nplants.2017.53

Creasey KM, Zhai J, Borges F, Van Ex F, Regulski M, Meyers BC, Martienssen RA (2014) miRNAs trigger widespread epigenetically activated siRNAs from transposons in Arabidopsis. Nature 508:411-415. https://doi.org/10.1038/nature13069

Cui J, Luan Y, Jiang N, Bao H, Meng J (2017) Comparative transcriptome analysis between resistant and susceptible tomato allows the identification of lncRNA16397 conferring resistance to Phytophthora infestans by co-expressing glutaredoxin. Plant J 89:577-589. https://doi.org/10.1111/tpj.13408

Das SS, Karmakar P, Nandi AK, Sanan-Mishra N (2015) Small RNA mediated regulation of seed germination. Front Plant Sci 6:828. https://doi.org/10.3389/fpls.2015.00828

De Paola D, Zuluaga DL, Sonnante G (2016) The miRNAome of durum wheat: isolation and characterization of conserved and novel microRNAs and their target genes. BMC Genomics 17:505. https://doi.org/10.1186/s12864-016-2838-4

Deng P, Muhammad S, Cao M, Wu L (2018) Biogenesis and regulatory hierarchy of phased small interfering RNAs in plants. Plant Biotechnol J 16:965-975. https://doi.org/10.1111/pbi.12882

Di C, Yuan J, Wu Y, Li J, Lin H, Hu L, Zhang T, Qi Y, Gerstein MB, Guo Y, Lu ZJ (2014) Characterization of stress-responsive IncRNAs in Arabidopsis thaliana by integrating expression, epigenetic and structural features. Plant J 80:848-861. https://doi. org/10.1111/tpj.12679

Ding J, Shen J, Mao H, Xie W, Li X, Zhang Q (2012) RNA-directed DNA methylation is involved in regulating photoperiod-sensitive male sterility in rice. Mol Plant 5:1210-1216. https://doi.org/10. 1093/mp/sss095

Ding Z, Tie W, Fu L, Yan Y, Liu G, Yan W, Li Y, Wu C, Zhang J, $\mathrm{Hu}$ W (2019) Strand-specific RNA-seq based identification and functional prediction of drought-responsive lncRNAs in cassava. BMC Genomics 20:214. https://doi.org/10.1186/ s12864-019-5585-5

Fabbri M, Girnita L, Varani G, Calin GA (2019) Decrypting noncoding RNA interactions, structures, and functional networks. Genome Res 29:1377-1388. https://doi.org/10.1101/gr.247239.118

Fan J, Quan W, Li GB, Hu XH, Wang Q, Wang H, Li XP, Luo X, Feng Q, Hu ZJ, Feng H (2020) circRNAs are involved in the riceMagnaporthe oryzae interaction. Plant Physiol 182:272-286. https://doi.org/10.1104/pp.19.00716

Fan Y, Yang J, Mathioni SM, Yu J, Shen J, Yang X, Wang L, Zhang Q, Cai Z, Xu C, Li X (2016) PMS1T, producing phased smallinterfering RNAs, regulates photoperiod-sensitive male sterility in rice. Proc Nat Acad Sci USA 113:15144-15149. https://doi. org/10.1073/pnas.1619159114

Fei Q, Xia R, Meyers BC (2013) Phased, secondary, small interfering RNAs in posttranscriptional regulatory networks. Plant Cell 25:2400-2415. https://doi.org/10.1105/tpc.113.114652

Ferdous J, Hussain SS, Shi BJ (2015) Role of microRNAs in plant drought tolerance. Plant Biotechnol J 13:293-305. https://doi. org/10.1111/pbi.12318

Ferdous J, Sanchez-Ferrero JC, Langridge P, Milne L, Chowdhury J, Brien C, Tricker PJ (2017) Differential expression of microRNAs and potential targets under drought stress in barley. Plant Cell Environ 40:11-24. https://doi.org/10.1111/pce.12764

Gleeson M, Constantin M, Carroll B, Mitter N (2014) MicroRNAs as regulators of adventitious root development. J Plant Biochem Biotechnol 23:339-347. https://doi.org/10.1007/ s13562-014-0269-3

Guan Q, Lu X, Zeng H, Zhang Y, Zhu J (2013) Heat stress induction of miR398 triggers a regulatory loop that is critical for 
thermotolerance in Arabidopsis. Plant J 74:840-851. https://doi. org/10.1111/tpj.12169

Guan X, Pang M, Nah G, Shi X, Ye W, Stelly DM, Chen ZJ (2014) miR828 and miR858 regulate homoeologous MYB2 gene functions in Arabidopsis trichome and cotton fibre development. Nat Commun 5:1-14. https://doi.org/10.1038/ncomms4050

Hackenberg M, Huang PJ, Huang CY, Shi BJ, Gustafson PE, Langridge PE (2013) A comprehensive expression profile of microRNAs and other classes of non-coding small RNAs in barley under phosphorous-deficient and sufficient conditions. DNA Res 20:109-125. https://doi.org/10.1093/dnares/dss037

Hajheidari M, Farrona S, Huettel B, Koncz Z, Koncz C (2012) $\mathrm{CDKF} ; 1$ and CDKD protein kinases regulate phosphorylation of serine residues in the C-terminal domain of Arabidopsis RNA polymerase II. Plant Cell 24:1626-1642. https://doi.org/ 10.1105/tpc.112.096834

He J, Jiang Z, Gao L, You C, Ma X, Wang X, Xu X, Mo B, Chen X, Liu L (2019) Genome-wide transcript and small RNA profiling reveals transcriptomic responses to heat stress. Plant Physiol 81:609-629. https://doi.org/10.1104/pp.19.00403

Holley RW, Apgar J, Everett GA, Madison JT, Marquisee M, Merrill SH, Penswick JR, Zamir A (1965) Structure of a ribonucleic acid. Science 147:1462-1465

Hou Y, Zhai Y, Feng L, Karimi HZ, Rutter BD, Zeng L, Choi DS, Zhang B, Gu W, Chen X, Ye W, Innes RW, Zhai J, Ma W (2019) A Phytophthora effector suppresses trans-kingdom RNAi to promote disease susceptibility. Cell Host Microbe 25:153-165. https://doi.org/10.1016/j.chom.2018.11.007

Hsieh LC, Lin SI, Kuo HF, Chiou TJ (2010) Abundance of tRNAderived small RNAs in phosphate-starved Arabidopsis roots. Plant Signal Behav 5:537-539. https://doi.org/10.4161/psb. 11029

Hua Y, Zhang C, Shi W, Chen H (2019) High-throughput sequencing reveals microRNAs and their targets in response to drought stress in wheat (Triticum aestivum L.). Biotechnol Biotechnol Equip 33:465-471. https://doi.org/10.1080/13102818.2019. 1586586

Jabnoune M, Secco D, Lecampion C, Shu Q, Poirier Y (2013) A rice cis-natural antisense RNA acts as a translational enhancer for its cognate mRNA and contributes to phosphate homeostasis and plant fitness. Plant Cell 25:4166-4182. https://doi.org/10. 1105/tpc.113.116251

Jacobs TB, LaFayette PR, Schmitz RJ, Parrott WA (2015) Targeted genome modifications in soybean with CRISPR/ Cas9. BMC Biotechnol 15:1-10. https://doi.org/10.1186/ s12896-015-0131-2

Jeong DH, Thatcher SR, Brown RS, Zhai J, Park S, Rymarquis LA, Meyers BC, Green PJ (2013) Comprehensive investigation of microRNAs enhanced by analysis of sequence variants, expression patterns, ARGONAUTE loading, and target cleavage. Plant Physiol 162:1225-1245. https://doi.org/10.1104/pp. 113.219873

Jiao Y, Wang Y, Xue D, Wang J, Yan M, Liu G, Dong G, Zeng D, Lu Z, Zhu X, Qian Q (2010) Regulation of OsSPL14 by OsmiR156 defines ideal plant architecture in rice. Nat Genet 42:541-544. https://doi.org/10.1038/ng.591

Karlik E, Gozukirmizi N (2018) Expression analysis of lncRNA AK370814 involved in the barley vitamin B6 salvage pathway under salinity. Mol Biol Rep 45:1597-1609. https://doi.org/10. 1007/s11033-018-4289-2

Katiyar-Agarwal S, Jin H (2010) Role of small RNAs in host-microbe interactions. Annu Rev Phytopath 48:225. https://doi.org/10. 1146/annurev-phyto-073009-114457

Khraiwesh B, Zhu JK, Zhu J (2012) Role of miRNAs and siRNAs in biotic and abiotic stress responses of plants. Biochim Biophys
Acta 1819:137-148. https://doi.org/10.1016/j.bbagrm.2011. 05.001

Lee RC, Feinbaum RL, Ambros V (1993) The C. elegans heterochronic gene lin-4 encodes small RNAs with antisense complementarity to lin-14. Cell 75:843-854. https://doi.org/10.1016/ 0092-8674(93)90529-Y

Li C, Zhang B (2016) MicroRNAs in control of plant development. J Cellular Physiol 231:303-313. https://doi.org/10.1002/jcp. 25125

Li SX, Liu JX, Liu ZY, Li X, Wu F, He Y (2014a) Heat-induced TAS1 target 1 mediates thermotolerance via heat stress transcription factorA1a-directed pathways in Arabidopsis. Plant Cell 26:1764-1780. https://doi.org/10.1105/tpc.114.124883

Li WX, Oono Y, Zhu J, He XJ, Wu JM, Iida K, Lu XY, Cui X, Jin H, Zhu JK (2008) The Arabidopsis NFYA5 transcription factor is regulated transcriptionally and post transcriptionally to promote drought resistance. Plant Cell 20:2238-2251. https://doi.org/10. 1105/tpc.108.059444

Liu T, Zhang L, Chen G, Shi T (2017a) Identifying and characterizing the circular RNAs during the lifespan of Arabidopsis leaves. Front Plant Sci 8:1278. https://doi.org/10.3389/fpls.2017.01278

Liu Y, El-Kassaby Y (2017) Regulatory cross-talk between microRNAs and hormone signalling cascades controls phenotypical variations: a case study in seed dormancy modulations during seed set of Arabidopsis thaliana. Plant Cell Rep 36:705-717. https:// doi.org/10.1007/s00299-017-2111-6

Lu T, Cui L, Zhou Y, Fan D, Gong H, Zhao Q, Zhou C, Zhao Y, Lu D, Luo J (2015) Transcriptome-wide investigation of circular RNAs in rice. RNA 21:2076-2087. https://doi.org/10.1261/rna. 052282.115

Lu X, Guan Q, Zhu J (2013) Downregulation of CSD2 by a heatinducible miR398 is required for thermotolerance in Arabidopsis. Plant Signal Behav 8:e24952. https://doi.org/10.4161/psb.24952

Luo R, Cao R, Jiao LY, Zhong M, Tang S, Wei X, Hu P (2018) The involvement of long non-coding RNAs in the formation of high temperature-induced grain chalkiness in rice. Plant Growth Regul 86:263-271. https://doi.org/10.1007/s10725-018-0426-0

Ma J, Zhao P, Liu S, Yang Q, Guo H (2020) The control of developmental phase transitions by microRNAs and their targets in seed plants. Int J Mol Sci 21:1971. https://doi.org/10.3390/ijms2 1061971

Mangrauthia SK, Bhogireddy S, Agarwal S, Prasanth VV, Voleti SR, Neelamraju S, Subrahmanyam D (2017a) Genome-wide changes in microRNA expression during short and prolonged heat stress and recovery in contrasting rice cultivars. J Exp Bot 68:23992412. https://doi.org/10.1093/jxb/erx111

Mangrauthia SK, Maliha A, Prathi NB, Marathi B (2017b) MicroRNAs: potential target for genome editing in plants for traits improvement. Ind J Plant Physiol 22:530-548. https://doi.org/ 10.1007/s40502-017-0326-8

Mangrauthia SK, Sailaja B, Pusuluri M, Jena B, Prasanth VV, Agarwal S, Senguttuvel P, Sarla N, Babu VR, Subrahmanyam D, Voleti SR (2018) Deep sequencing of small RNAs reveals ribosomal origin of microRNAs in Oryza sativa and their regulatory role in high temperature. Gene Rep 7:270-278. https://doi.org/10. 1016/j.genrep.2018.05.002

Martin RC, Asahina M, Liu PP, Kristof JR, Coppersmith JL, Pluskota WE, Bassel GW, Goloviznina NA, Nguyen TT, Martínez-Andújar C, Kumar MB (2010) The microRNA156 and microRNA172 gene regulation cascades at post-germinative stages in Arabidopsis. Seed Sci Res 20:79-87. https://doi.org/10.1017/S0960 258510000085

Martinez G, Choudury SG, Slotkin RK (2017) tRNA-derived small RNAs target transposable element transcripts. Nucleic Acids Res 45:5142-5152. https://doi.org/10.1093/nar/gkx103 
Mattick JS, Makunin IV (2006) Non-coding RNA. Hum Mol Genet 15:17-29. https://doi.org/10.1093/hmg/dd1046

Matzke MA, Kanno T, Matzke AJ (2015) RNA-directed DNA methylation: The evolution of a complex epigenetic pathway in flowering plants. Annu Rev Plant Biol 66:243-267. https://doi.org/10.1146/ annurev-arplant-043014-114633

Megha S, Basu U, Kav NN (2018) Regulation of low temperature stress in plants by microRNAs. Plant Cell Environ 41:1-15. https://doi. org/10.1111/pce.12956

Meng Y, Shao C (2012) Large-scale identification of mirtrons in Arabidopsis and rice. PLoS One 7:e31163. https://doi.org/10.1371/ journal.pone. 0031163

Morris KV, Mattick JS (2014) The rise of regulatory RNA. Nat Rev Genet 15:423-437. https://doi.org/10.1038/nrg3722

Navarro L, Dunoyer P, Jay F, Arnold B, Dharmasiri N, Estelle M, Voinnet O, Jones JD (2006) A plant miRNA contributes to antibacterial resistance by repressing auxin signaling. Science 312:436-439. https://doi.org/10.1126/science.1126088

Nejat N, Mantri N (2017) Emerging roles of long non-coding RNAs in plant response to biotic and abiotic stresses. Criti Rev Biotechnol 38:93-105. https://doi.org/10.1080/07388551.2017.1312270

Nosaka M, Itoh JI, Nagato Y, Ono A, Ishiwata A, Sato Y (2012) Role of transposon-derived small RNAs in the interplay between genomes and parasitic DNA in rice. PLoS Genet 8:e1002953. https://doi.org/10.1371/journal.pgen.1002953

Palazzo AF, Lee ES (2015) Non-coding RNA: what is functional and what is junk? Front Genet 6:2. https://doi.org/10.3389/fgene. 2015.00002

Pang J, Zhang X, Ma X, Zhao J (2019) Spatio-temporal transcriptional dynamics of maize long non-coding RNAs responsive to drought stress. Genes 10:138. https://doi.org/10.3390/genes10020138

Qin T, Zhao H, Cui P, Albesher N, Xiong L (2017) A nucleus-localized long non-coding RNA enhances drought and salt stress tolerance. Plant Physiol 175:1321-1336. https://doi.org/10.1104/pp. 17.00574

Ravichandran S, Ragupathy R, Edwards T, Domaratzki M, Cloutier $S$ (2019) MicroRNA-guided regulation of heat stress response in wheat. BMC Genomics 20:488. https://doi.org/10.1186/ s12864-019-5799-6

Reyes JL, Chua NH (2007) ABA induction of miR159 controls transcript levels of two MYB factors during Arabidopsis seed germination. Plant J 49:592-606. https://doi.org/10.1111/j.1365-313X. 2006.02980

Rogers K, Chen X (2013) Biogenesis, turnover, and mode of action of plant microRNAs. Plant Cell 25:2383-2399. https://doi.org/10. 1105/tpc. 113.113159

Rosa C, Kuo YW, Wuriyanghan H, Falk BW (2018) RNA interference mechanisms and applications in plant pathology. Annu Rev Phytopathol 56:581-610. https://doi.org/10.1146/annur ev-phyto-080417-050044

Sablok G, Zhao H, Sun X (2016) Plant circular RNAs (circRNAs): transcriptional regulation beyond miRNAs in plants. Mol Plant 9:192-194. https://doi.org/10.1016/j.molp.2015.12.021

Sailaja B, Anjum N, Vishnu prashanth V, Sarla N, Subrahmanyam D, Voleti SR, Viraktamath BC, Mangrauthia SK (2014) Comparative study of susceptible and tolerant genotype reveals efficient recovery and root system contributes to heat stress tolerance in rice. Plant Mol Biol Rep 32:1228-1240. https://doi.org/10.1007/ s11105-014-0728-y

Salvador-Guirao R, Baldrich P, Weigel D, Rubio-Somoza I, San Segundo B (2018) The microRNA miR773 is involved in the Arabidopsis immune response to fungal pathogens. Mol Plant Microbe Interact 31:249-259. https://doi.org/10.1094/ MPMI-05-17-0108-R

Shuai P, Liang D, Tang S, Zhang Z, Ye CY, Su Y, Xia X, Yin W (2014) Genome-wide identification and functional prediction of novel and drought-responsive lincRNAs in Populus trichocarpa. J Exp Bot 65:4975-4983. https://doi.org/10.1093/jxb/eru256

Shuai P, Su Y, Liang D, Zhang Z, Xia X, Yin W (2016) Identification of phasiRNAs and their drought- responsiveness in Populus trichocarpa. FEBS Lett 590:3616-3627. https://doi.org/10.1002/ 1873-3468.12419

Sobkowiak L, Karlowski W, Jarmolowski A, Szweykowska-Kulinska Z (2012) Non-canonical processing of Arabidopsis pri-miR319a/ $\mathrm{b} / \mathrm{c}$ generates additional microRNAs to target one RAP2.12 mRNA isoform. Front Plant Sci 3:46. https://doi.org/10.3389/ fpls.2012.00046

Son DJ, Kumar S, Takabe W, Kim CW, Ni CW, Alberts-Grill N, Jang IH, Kim S, Kim W, Kang SW, Baker AH (2013) The atypical mechanosensitive microRNA-712 derived from pre-ribosomal RNA induces endothelial inflammation and atherosclerosis. Nat Commun 4:3000. https://doi.org/10.1038/ncomms4000

Song JB, Gao S, Wang Y, Li BW, Zhang YL, Yang ZM (2016) miR394 and its target gene LCR are involved in cold stress response in Arabidopsis. Plant Gene 5:56-64. https://doi.org/10.1016/j. plgene.2015.12.001

Song X, Li Y, Cao X, Qi Y (2019) MicroRNAs and their regulatory roles in plant-environment interactions. Annu Rev Plant Biol 70:489-525. https://doi.org/10.1146/annurev-arpla nt-050718-100334

Swarup R, Denyer T (2018) miRNAs in plant development. Annu Plant Rev Ol 2:689-712. https://doi.org/10.1002/9781119312 994.apr0649

Taft RJ, Glazov EA, Lassmann T, Hayashizaki Y, Carninci P, Mattick JS (2009) Small RNAs derived from snoRNAs. RNA 15:12331240. https://doi.org/10.1261/rna.1528909

Tang J, Chu C (2017) MicroRNAs in crop improvement: fine-tuners for complex traits. Nat Plants 3:17077. https://doi.org/10.1038/ nplants.2017.77

Wang T, Chen L, Zhao M, Tian Q, Zhang WH (2011) Identification of drought-responsive microRNAs in Medicago truncatula by genome-wide high-throughput sequencing. BMC Genomics 12:367. https://doi.org/10.1186/1471-2164-12-367

Wang S, Wu K, Yuan Q, Liu X, Liu Z, Lin X, Zeng R, Zhu H, Dong G, Qian Q, Zhang G (2012) Control of grain size, shape and quality by OsSPL16 in rice. Nat Genet 44:950-954. https://doi. org/10.1038/ng.2327

Wang C, Ye J, Tang W, Liu Z, Zhu C, Wang M, Wan J (2013) Loop nucleotide polymorphism in a putative miRNA precursor associated with seed length in rice (Oryza sativa L.). Int J Biol Sci 9:578-586. https://doi.org/10.7150/ijbs.6357

Wang Y, Fan X, Lin F, He G, Terzaghi W, Zhu D, Deng XW (2014) Arabidopsis noncoding RNA mediates control of photomorphogenesis by red light. Proc Natl Acad Sci U S A 111:1035910364. https://doi.org/10.1073/pnas.1409457111

Wang X, Ai G, Zhang C, Cui L, Wang J, Li H, Zhang J, Ye Z (2015a) Expression and diversification analysis reveals transposable elements play important roles in the origin of Lycopersicon-specific lncRNAs in tomato. New Phytol 209:1442-1455. https://doi.org/ 10.1111/nph.13718

Wang TZ, Liu M, Zhao MG, Chen R, Zhang WH (2015b) Identification and characterization of long noncoding RNAs involved in osmotic and salt stress in Medicago truncatula using genomewide high-throughput sequencing. BMC Plant Biol 15:131. https://doi.org/10.1186/s12870-015-0530-5

Wang Y, Yang M, Wei S, Qin F, Zhao H, Suo B (2016a) Identification of circular RNAs and their targets in leaves of Triticum aestivum L. under dehydration stress. Front Plant Sci 7:2024. https://doi. org/10.3389/fpls.2016.02024

Wang Y, Li H, Sun Q, Yao Y (2016b) Characterization of small RNAs derived from tRNAs, rRNAs and snoRNAs and their response to 
heat stress in wheat seedlings. PLoS One 11:e0150933. https:// doi.org/10.1371/journal.pone.0150933

Wang H, Jiao X, Kong X, Hamera S, Wu Y, Chen X, Fang R, Yan $\mathrm{Y}$ (2016c) A signaling cascade from miR444 to RDR1 in rice antiviral RNA silencing pathway. Plant Physiol 170:23652377. https://doi.org/10.1104/pp.15.01283

Wang Z, Liu Y, Li D, Li L, Zhang Q, Wang S, Huang H (2017a) Identification of circular RNAs in kiwifruit and their speciesspecific response to bacterial canker pathogen invasion. Front Plant Sci 8:413. https://doi.org/10.3389/fpls.2017.00413

Wang D, Qu Z, Yang L, Zhang Q, Liu ZH, Do T, Adelson DL, Wang ZY, Searle I, Zhu JK (2017b) Transposable elements (TEs) contribute to stress-related long intergenic noncoding RNAs in plants. Plant J 90:133-146. https://doi.org/10.1111/tpj.13481

Wang Y, Luo X, Sun F, Hu J, Zha X, Su W, Yang J (2018a) Overexpressing lncRNA LAIR increases grain yield and regulates neighbouring gene cluster expression in rice. Nat Commun 9:3516. https://doi.org/10.1038/s41467-018-05829-7

Wang J, Lin J, Wang H, Li X, Yang Q, Li H, Chang Y (2018b) Identification and characterization of circRNAs in Pyrus betulifolia Bunge under drought stress. PLoS One 13:e0200692. https:// doi.org/10.1371/journal.pone.0200692

Wang J, Mei J, Ren G (2019a) Plant microRNAs: biogenesis, homeostasis, and degradation. Front Plant Sci 10:360. https://doi.org/ 10.3389/fpls.2019.00360

Wang A, Hu J, Gao C, Chen G, Wang B, Lin C, Song L, Ding Y, Zhou G (2019b) Genome-wide analysis of long non-coding RNAs unveils the regulatory roles in the heat tolerance of Chinese cabbage (Brassica rapa ssp. chinensis). Sci Rep 9:5002. https://doi.org/10.1038/s41598-019-41428-2

Wang P, Dai L, Ai J, Wang Y, Ren F (2019c) Identification and functional prediction of cold-related long non-coding RNA (lncRNA) in grapevine. Sci Rep 9:6638. https://doi.org/10. 1038/s41598-019-43269-5

Weidong QI, Hongping CH, Zuozhen YA, Biaolin HU, Xiangdong L, Bing A, Yuan L, Yu H, Jiankun X, Fantao Z (2020) Systematic characterization of long non-coding RNAs and their responses to drought stress in Dongxiang wild rice. Rice Sci 27:21-31

Whittaker C, Dean C (2017) The FLC locus: a platform for discoveries in epigenetics and adaptation. Annu Rev Cell Dev Biol 33:555-575. https://doi.org/10.1146/annurev-cellb io-100616-060546

Wierzbicki AT, Haag JR, Pikaard CS (2008) Noncoding transcription by RNA polymerase Pol IVb/Pol V mediates transcriptional silencing of overlapping and adjacent genes. Cell 135:635-648. https://doi.org/10.1016/j.cell.2008.09.035

Wierzbicki AT, Ream TS, Haag JR, Pikaard CS (2009) RNA polymerase V transcription guides ARGONAUTE4 to chromatin. Nature Genet 41:630-634. https://doi.org/10.1038/ng.365

Wu H, Li B, Iwakawa HO, Pan Y, Tang X, Ling-Hu Q, Liu Y, Sheng S, Feng L, Zhang H, Zhang X, Guo H (2020) Plant 22-nt siRNAs mediate translational repression and stress adaptation. Nature 581:89-93. https://doi.org/10.1038/s41586-020-2231-y

Xia R, Xu J, Meyers B (2017) The emergence, evolution, and diversification of the miR390-TAS3-ARF pathway in land plants. Plant Cell 29:1232-1247. https://doi.org/10.1105/tpc.17.0018

Xie F, Wang Q, Zhang B (2015) Global microRNA modification in cotton (Gossypium hirsutum L.). Plant Biotechnol J 13:492500. https://doi.org/10.1111/pbi.12271

Yang K, Wen X, Mudunuri S, Varma GS, Sablok G (2019) Diff isomiRs: Large-scale detection of differential isomiRs for understanding non-coding regulated stress omics in plants. Sci Rep 9:1-12. https://doi.org/10.1038/s41598-019-38932-w

Yu X, Wang H, Lu Y, de Ruiter M, Cariaso M, Prins M, van Tunen A, He Y (2012) Identification of conserved and novel microRNAs that are responsive to heat stress in Brassica rapa. J Exp Bot 63:1025-1038. https://doi.org/10.1093/jxb/err337

Yu Y, Zhang Y, Chen X, Chen Y (2019) Plant noncoding RNAs: hidden players in development and stress responses. Annu Rev Cell Dev Biol 35:407-431. https://doi.org/10.1146/annurev-cellb io- $100818-125218$

Zhang B, Wang Q (2016) MicroRNA, a new target for engineering new crop cultivars. Bioengineered 7:7-10. https://doi.org/10. 1080/21655979.2016.1141838

Zhang F, Luo X, Zhou Y, Xie J (2016b) Genome-wide identification of conserved microRNA and their response to drought stress in dongxiang wild rice (Oryza rufipogon Griff.). Biotechnol Lett 38:711-721. https://doi.org/10.1007/s10529-015-2012-0

Zhang N, Zhang D, Chen SL, Gong BQ, Guo Y, Xu L, Zhang XN, Li JF (2018a) Engineering artificial microRNAs for multiplex gene silencing and simplified transgenic screen. Plant Physiol 178:989-1001. https://doi.org/10.1104/pp.18.00828

Zhang P, Li S, Chen M (2020) Characterization and function of circular RNAs in plants. Front Mol Biosci 7:91. https://doi.org/10.3389/ fmolb.2020.00091

Zhang S, Sun L, Kragler F (2009) The phloem-delivered RNA pool contains small noncoding RNAs and interferes with translation. Plant Physiol 150:378-387. https://doi.org/10.1104/pp.108. 134767

Zhang X, Zou Z, Gong P, Zhang J, Ziaf K, Li H, Xiao F, Ye Z (2011a) Over-expression of microRNA169 confers enhanced drought tolerance to tomato. Biotechnol Lett 33:403-409. https://doi.org/10. 1007/s10529-010-0436-0

Zhang XO, Dong R, Zhang Y, Zhang JL, Luo Z, Zhang J, Chen LL, Yang L (2016a) Diverse alternative back-splicing and alternative splicing landscape of circular RNAs. Genome Res 26:12771287. https://doi.org/10.1101/gr.202895.115

Zhang YC, Yu Y, Wang CY, Li ZY, Liu Q, Xu J, Liao JY, Wang XJ, Qu LH, Chen F, Xin P (2013a) Overexpression of microRNA osmiR397 improves rice yield by increasing grain size and promoting panicle branching. Nat Biotechnol 31:848-852. https:// doi.org/10.1038/nbt.2646

Zhao J, He Q, Chen G, Wang L, Jin B (2016) Regulation of non-coding RNAs in heat stress responses of plants. Front Plant Sci 7:1213. https://doi.org/10.3389/fpls.2016.01213

Zhao W, Chu S, Jiao Y (2019) Present scenario of circular RNAs (circRNAs) in plants. Front Plant Sci 10:379. https://doi.org/10. 3389/fpls.2019.00379

Zheng C, Ye M, Sang M, Wu R (2019a) A regulatory network for miR156-SPL module in Arabidopsis thaliana. Int J Mol Sci 20:6166. https://doi.org/10.3390/ijms20246166

Zhou B, Leng J, Ma Y, Fan P, Li Y, Yan H, Xu Q (2020) BrmiR828 targets Brpap1, Brmyb82, and Brtas4 involved in the light induced anthocyanin biosynthetic pathway in Brassica rapa. Int J Mol Sci 21:4326. https://doi.org/10.3390/ijms21124326

Zhou J, Deng K, Cheng Y, Zhong Z, Tian L, Tang X, Tang A, Zheng X, Zhang T, Qi Y, Zhang Y (2017) CRISPR-Cas9 based genome editing reveals new insights into microRNA function and regulation in rice. Front Plant Sci 8:1598. https://doi.org/10.3389/fpls. 2017.01598

Zhou M, Law JA (2015) RNA Pol IV and V in gene silencing: rebel polymerases evolving away from Pol II's rules. Curr Opin Plant Biol 27:154-164. https://doi.org/10.1016/j.pbi.2015.07.005

Zhou M, Li D, Li Z, Hu Q, Yang C, Zhu L, Luo H (2013) Constitutive expression of a miR319 gene alters plant development and enhances salt and drought tolerance in transgenic creeping bentgrass. Plant Physiol 161:1375-1391. https://doi.org/10.1104/pp. 112.208702

Zhou R, Zhu Y, Zhao J, Fang Z, Wang S, Yin J, Chu Z, Ma D (2018) Transcriptome-wide identification and characterization of potato circular RNAs in response to Pectobacterium carotovorum 
Subspecies brasiliense Infection. Int J Mol Sci 19:71. https:// doi.org/10.3390/ijms19010071

Zhu L, Ow DW, Dong Z (2018) Transfer RNA-derived small RNAs in plants. Sci China Life Sci 61:155-161. https://doi.org/10.1007/ s11427-017-9167-5
Publisher's note Springer Nature remains neutral with regard to jurisdictional claims in published maps and institutional affiliations. 